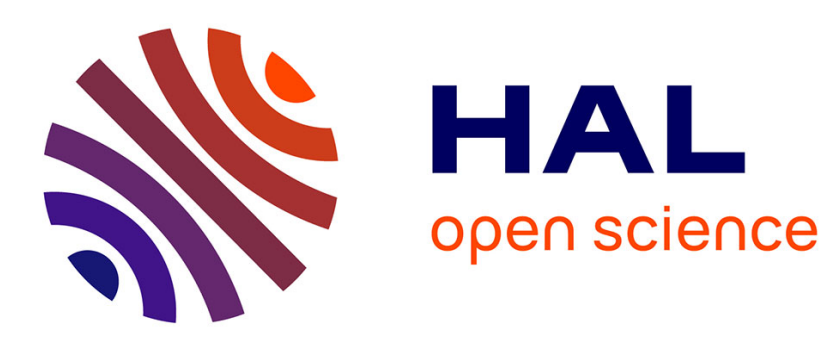

\title{
Larval Zebrafish Use Olfactory Detection of Sodium and Chloride to Avoid Salt Water
}

Kristian J Herrera, Thomas Panier, Drago Guggiana-Nilo, Florian Engert

\section{To cite this version:}

Kristian J Herrera, Thomas Panier, Drago Guggiana-Nilo, Florian Engert. Larval Zebrafish Use Olfactory Detection of Sodium and Chloride to Avoid Salt Water. Current Biology - CB, 2021, 31 (4), pp.782-793.e3. 10.1016/j.cub.2020.11.051 . hal-03197695

\section{HAL Id: hal-03197695 \\ https://hal.sorbonne-universite.fr/hal-03197695}

Submitted on 14 Apr 2021

HAL is a multi-disciplinary open access archive for the deposit and dissemination of scientific research documents, whether they are published or not. The documents may come from teaching and research institutions in France or abroad, or from public or private research centers.
L'archive ouverte pluridisciplinaire HAL, est destinée au dépôt et à la diffusion de documents scientifiques de niveau recherche, publiés ou non, émanant des établissements d'enseignement et de recherche français ou étrangers, des laboratoires publics ou privés. 


\section{Current Biology \\ Larval Zebrafish Use Olfactory Detection of Sodium and Chloride to Avoid Salt Water}

\section{Highlights}

- Zebrafish respond to and avoid increasing salt concentrations

- The activity of a sparse subset of olfactory neurons reflects external salinity

- Removal of olfactory salt sensitivity also eliminates behavioral responses to $\mathrm{NaCl}$

- Olfactory salt detection is sensitive to both cation and anion identities

\section{Authors}

Kristian J. Herrera, Thomas Panier, Drago Guggiana-Nilo, Florian Engert

Correspondence

kjherrera23@gmail.com

\section{In Brief}

In this study, Herrera et al. examine the behavioral and sensory responses of larval zebrafish, a fresh-water species, to salt. They find that larvae strongly broadly avoid any increases in environmental salinity and that olfactory detection of both cation and anions likely drives this behavior. 


\title{
Larval Zebrafish Use Olfactory Detection of Sodium and Chloride to Avoid Salt Water
}

\author{
Kristian J. Herrera, ${ }^{1,4, *}$ Thomas Panier, ${ }^{2}$ Drago Guggiana-Nilo, ${ }^{3}$ and Florian Engert ${ }^{1}$ \\ 1Department of Molecular and Cellular Biology, Harvard University, 16 Divinity Avenue, Cambridge, MA 02138, USA \\ 2Laboratoire Jean Perrin (LJP), Institut de Biologie Paris-Seine (IBPS), Sorbonne Université, CNRS, 4 Place Jussieu, 75005 Paris, France \\ ${ }^{3}$ Synapses - Circuits - Plasticity, Max Planck Institute for Neurobiology, Munich, Germany \\ ${ }^{4}$ Lead Contact \\ *Correspondence: kjherrera23@gmail.com \\ https://doi.org/10.1016/j.cub.2020.11.051
}

\section{SUMMARY}

Salinity levels constrain the habitable environment of all aquatic organisms. Zebrafish are freshwater fish that cannot tolerate high-salt environments and would therefore benefit from neural mechanisms that enable the navigation of salt gradients to avoid high salinity. Yet zebrafish lack epithelial sodium channels, the primary conduit land animals use to taste sodium. This suggests fish may possess novel, undescribed mechanisms for salt detection. In the present study, we show that zebrafish indeed respond to small temporal increases in salt by reorienting more frequently. Further, we use calcium imaging techniques to identify the olfactory system as the primary sense used for salt detection, and we find that a specific subset of olfactory receptor neurons encodes absolute salinity concentrations by detecting monovalent anions and cations. In summary, our study establishes that zebrafish larvae have the ability to navigate and thus detect salinity gradients and that this is achieved through previously undescribed sensory mechanisms for salt detection.

\section{INTRODUCTION}

All organisms must maintain their internal ionic content within a tight window. Across vertebrates, the ion concentrations of plasma are largely conserved, with sodium and chloride ions present above $100 \mathrm{mM}$ and all other major ions kept below 5-10 mM. However, the mechanisms that animals utilize for osmoregulation are diverse and depend on their environment. Land animals and marine mammals balance the consumption of ion-rich food or liquids with the intake of ion-poor water and excretion of excess ions. ${ }^{1,2}$ On the other hand, fish and amphibians supplement fluid consumption and excretion with the function of a specialized system of epidermal cells, ionocytes, that exchange ions with their surrounding water. ${ }^{3,4}$ Critically, the direction and mechanism of ion exchange depend on the salinity of the animal's natural environment. While ionocytes of freshwater fish must acquire salts, those of marine fish excrete them.

Terrestrial animals encounter and balance salt through food intake, leading their gustatory systems to evolve high sensitivity to salt. ${ }^{5-8}$ They usually use two separate channels for discriminating either appetitive $(<100 \mathrm{mM})$ or aversive $(>150 \mathrm{mM}) \mathrm{NaCl}$ concentrations. In vertebrates, these are respectively mediated by epithelial sodium channels, ${ }^{5}$ which specifically allow sodium influx, ${ }^{9}$ and bitter taste receptors ${ }^{6}$ that are broadly gated by noxious stimulants. By contrast, the primary external salt sensors for fish are unknown at both the tissue and molecular level. However, aquatic animals are directly exposed to changes in environmental salinity, suggesting it might be advantageous for their salt detection to be integrated with a variety of modalities, beyond taste reception, that are suited for environmental navigation, such as olfaction, ${ }^{10}$ somatosensation, ${ }^{11}$ solitary chemosensors on the body ${ }^{12}$ and gills, ${ }^{13}$ or even the mechanosensory lateral line. ${ }^{14}$ The teleost gustatory system, although clearly implicated in feeding-related behaviors, is less likely to play a critical role in salt gradient navigation, because teleosts have lost all homologs of the mammalian epithelial sodium channel family ${ }^{15}$ and bitter taste receptors do not respond to the relevant salt concentrations. ${ }^{16}$ As such, it is still unclear which molecular mechanisms fish use to detect external $\mathrm{NaCl}$ and to appropriately adjust their behavior.

Zebrafish, in particular, would benefit from the ability to detect and navigate $\mathrm{NaCl}$ gradients because the environments in which they likely evolved are characterized by dramatic changes in local salinity levels: the river basins that surround the Ganges River in India and Bangladesh, for example, ${ }^{17,18}$ are characterized by soft, ion-poor water with $\mathrm{NaCl}$ concentrations as low as 1 part per trillion, which can increase locally by orders of magnitude during the dry season. ${ }^{19}$ Importantly, such changes lead to elevated stress and cortisol levels and are ultimately lethal, ${ }^{20-22}$ which makes neural mechanisms for detecting and avoiding salt gradients paramount for survival. Here, we show that zebrafish have evolved behavioral strategies to avoid highsalt environments and that this behavior is mediated by the olfactory system through a subset of olfactory sensory neurons that detect the combined presence of sodium and chloride.

\section{RESULTS}

Larval Zebrafish Avoid High-Salt Environments To test whether larval zebrafish avoid high-saline environments, we developed an assay for the detailed observation of larvae swimming in a salt gradient (Figure 1A; STAR Methods). 
A

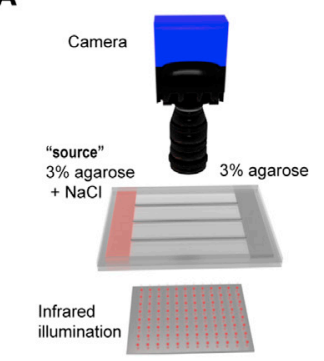

E

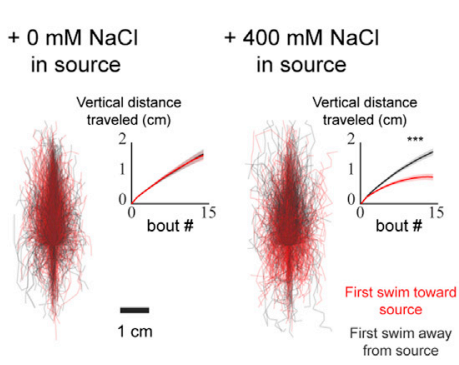

B

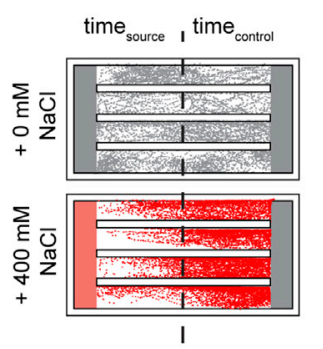

C

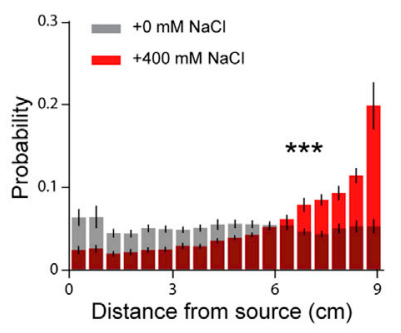

D

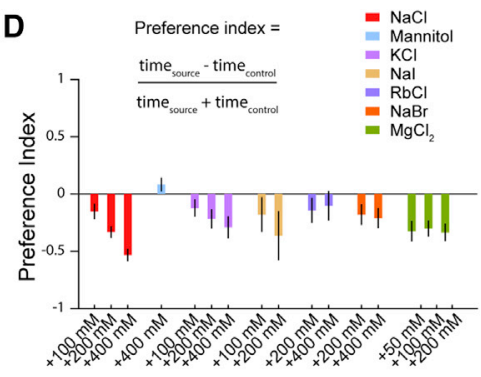

F

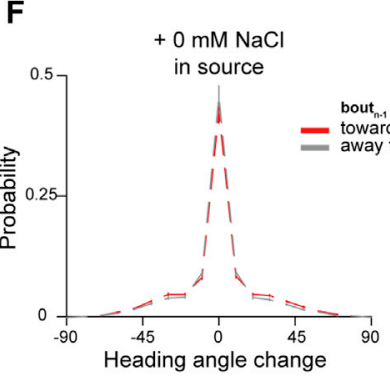

G

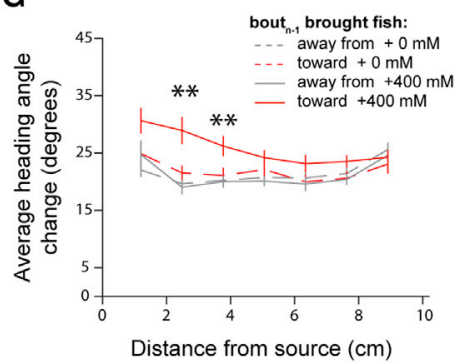

H

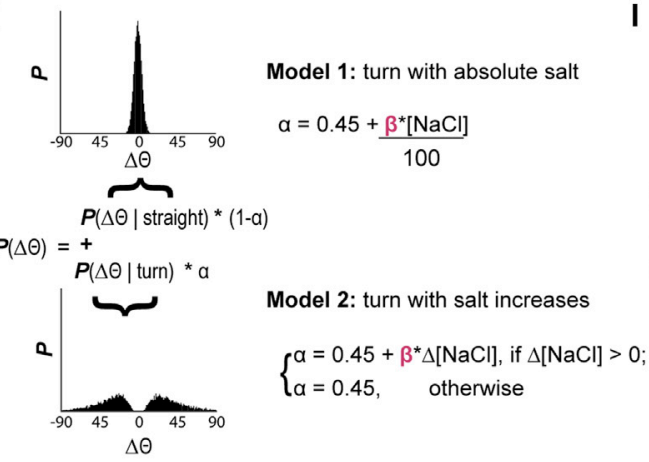

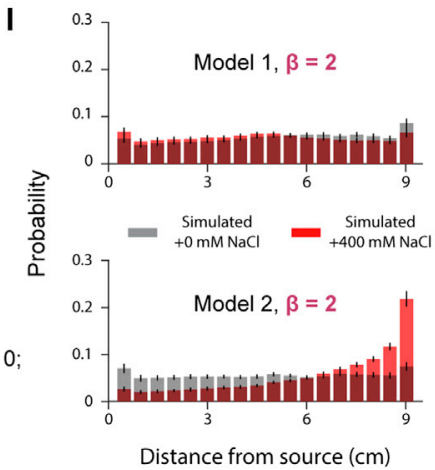

J

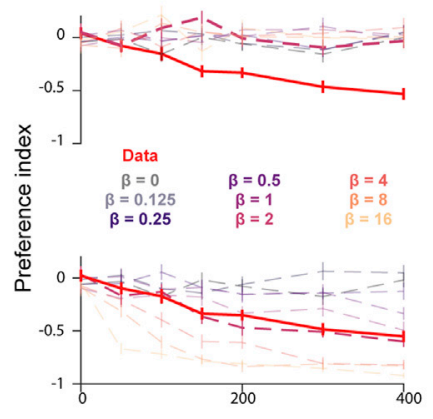

added $\mathrm{NaCl}$ concentration $(\mathrm{mM})$

Figure 1. Larval Zebrafish Avoid High-Salt Environments by Responding to Increases in Salinity (A) Schematic of the rig used to perform chemical place preference assays.

(B) Sample experiments when $0 \mathrm{mM}$ (top) or $400 \mathrm{mM}$ (bottom) are added to the source agarose. Individual dots demarcate position of larvae at every $50^{\text {th }}$ frame spanning the entire experiment.

(C) Histogram of positional occupancy over the entire experiment by larvae when the source gel contains either $0 \mathrm{mM}(n=30)$ or $400 \mathrm{mM} \mathrm{NaCl}(n=24)$ added (Mann-Whitney $U$ test; $p<0.001$ ) (error bars: mean \pm SEM across fish).

(D) Preference indices toward different chemical solutions (error bars: mean $\pm \mathrm{SEM}$ ).

(E) Individual trajectories of 15 bouts that follow a bout that climbs (red) or descends (gray) gradients with 0 (left) and $400 \mathrm{mM}$ (right) $\mathrm{NaCl}$ added to the source. Inset depicts average accumulated distance along the gradient axis in the direction of the first bout (two-sided t test; $p<0.001$ for total distance when $400 \mathrm{mM}$ in source; shading indicates SEM).

(F) Average turn angle as the larvae swims toward or away from the source as a function of their position in the arena (error bars: mean \pm SEM; Bonferronicorrected t test; $p<0.01$ ).

(G) Difference in average turn angle of bouts that follow an increased salt concentration compared to those that follow a decreased salt concentration for different concentrations of source salt (error bars: mean \pm SEM; Bonferroni-corrected t test; $p<0.01$ ).

(H) Description of the two models being simulated: larvae respond to absolute (model 1) or relative (model 2) salt concentrations.

(I) Spatial distribution of larvae in a simulated linear salt gradient when model 1 (top) or model 2 (bottom) are active (error bars: mean \pm SEM).

(J) Preference indices toward different concentrations of $\mathrm{NaCl}$ that result from simulating fish according to both algorithms for a range of salt sensitivities ( $\beta$ ) (error bars: mean \pm SEM).

See also Figure S1.

In this assay, four larvae are placed in separate lanes bookended by two agar pads made either from filtered fish water alone (control) or from fish water plus the salt being tested (source). We show that diffusion of sodium chloride $(\mathrm{NaCl})$ from the source generates a persistent salinity gradient throughout the lanes (Figure $\mathrm{S} 1 \mathrm{~A}$ ) and that larvae navigating such a gradient spend significantly more time away from the source than in control conditions where no salt is added (Figures $1 \mathrm{~B}$ and $1 \mathrm{C}$ ). This aversion emerges alongside the developing salt gradient over the first $15 \mathrm{~min}$ and is stable afterward (Figure S1B). This behavior is robust and demonstrates that larvae as young as 5 days post-fertilization (dpf) (Figure S1C) 


\section{Current Biology}

are already equipped with an active strategy to avoid regions of high salinity.

External sodium chloride fluctuations change several environmental parameters, such as osmolarity, net conductivity, and specific ion identities. Any of these may be detected and used by the larvae to avoid high salt, and in order to dissect the relevance of each parameter, we tested the preference of larvae to a series of compounds that isolate specific factors. For example, a general aversion to increased osmolarity was tested by generating gradients of a sugar alcohol, mannitol, that were equimolar with the previously tested $\mathrm{NaCl}$ gradients (Figure 1D). Under these conditions, the larvae exhibit no place preference. By contrast, zebrafish reliably avoided every ionic solution that we tested, whether it consisted of chloride paired with another monovalent $(\mathrm{KCl})$ or divalent cation $\left(\mathrm{MgCl}_{2}\right)$ or sodium paired with another anion (Nal). Compared to sodium chloride, larger ions (i.e., rubidium or bromide) were more weakly and inconsistently avoided by zebrafish larvae, though this may be the result of slower diffusion leading to lower concentration gradients. Overall, our observation that larvae indiscriminately avoid ionic solutions fails to tell us whether the mechanism underlying $\mathrm{NaCl}$ detection is based on a broad conductivity sensor or whether it is specific to different ions, as it is unclear whether or not the mechanisms responsible for avoiding these other ions belong to identical or parallel neural pathways. A detailed resolution of this question necessitates the identification of the relevant sensory neurons, the approach to which is described in later sections.

Salt Avoidance Is Driven by Detecting Salt Increases Next, we wished to understand the specific heuristics that larval zebrafish employ to avoid high-salt concentrations. We observed that, in all conditions, larvae predominantly swim back and forth between the two ends of the arena (Figure S1E), while aligned parallel to the longitudinal axis (Figure S1D). As larvae ascend a salt gradient, however, they reverse directions earlier than during gradient descent (Figure 1E). This suggests a biased random walk where increasing salt concentrations amplify the larva's reorientation frequency. ${ }^{23,24}$ To test this hypothesis, we compared the distribution of reorientation angles during bouts that follow a swim toward salt with those that follow a swim away from salt. With no gradient, larvae do not alter their turn statistics between these two cases (Figure 1F, left). By contrast, during gradient navigation, larvae are significantly more likely to execute a 20- to 40-degree turn if the previous bout brought them closer to the salt rather than away from it (Figure 1F, right). Compared to control conditions, turning magnitudes are only modulated by the salt concentration as the larva climbs the gradient (Figure $1 \mathrm{G}$ ), where the size of this modulation increases with higher salt levels in the source (Figure S1F), indicating salinity increases, rather than absolute concentrations, drive turning.

To test whether a biased random walk is sufficient to explain the larvae's avoidance behavior, we simulated the ability of virtual larvae utilizing natural swimming and turn statistics to navigate a salt gradient. We compared the performance of these simplified agents in conditions where turning was either upregulated as a function of absolute salinity levels or, alternatively, where turning probability increased after relative increases in salinity (Figure $1 \mathrm{H}$; see STAR Methods). We found that only the latter adequately captured the avoidance behavior of real animals (Figures $1 \mathrm{I}$ and $1 \mathrm{~J}$ ). A potential concern is that, as formulated, this model predicts that larvae would swim readily toward water with no salt at all, a counterintuitive result given the significantly deleterious effects this would have on the animals (Figure S1G). Testing this prediction with deionized water, we found, that larvae indeed swim toward the lowest salt concentrations (Figure $\mathrm{S} 1 \mathrm{H}$ ), which suggests that larvae may not seek an optimal external $\mathrm{NaCl}$ setpoint but instead always avoid increasing salinity. A possible explanation for this simplistic, if maladaptive, strategy is that regions of deleteriously low salinity are extremely rare and likely did not impart any selective pressure onto these animals.

We have so far identified that larval zebrafish avoid waters with high salinity and do so by responding to salt concentration increases. We next wished to identify the sensory modalities that detect salt. However, to identify such sensory regions, we wished to use calcium imaging techniques (i.e., light-sheet or two-photon point scanning microscopy) that require us to immobilize the animal's head. To accommodate this constraint, we designed a stimulation setup (Figure $2 \mathrm{~A}$ ) to rapidly and reversibly present different chemicals to the larva's face (Figures S2A-S2C) while the head and torso are embedded in agarose and the tail is free to move. We find that larvae in this preparation respond to salt pulses by vigorous tail flicks (Figure 2B) in a concentrationdependent manner (Figure 2C). Consistent with the free-swimming behavior, the larvae are most responsive during the onset of a $\mathrm{NaCl}$ pulse, corresponding to a recent concentration increase (Figure 2D). Therefore, we treat this preparation as a reasonable proxy for the more naturalistic condition of a larva swimming freely in a concentration gradient.

\section{Activity in the Olfactory and Lateral Line Systems Reflects External Salinity}

To screen for the brain regions most sensitive to such $\mathrm{NaCl}$ pulses, we combined our tethered preparation with a custombuilt light-sheet microscope (Figure 3A; Video S1). This allowed us to perform volumetric imaging across most of the brain in transgenic larvae expressing GCaMP6s under control of the pan-neuronal HuC (or elav/3) promoter (Figure 3B) ${ }^{25}$ while delivering pulses of different $\mathrm{NaCl}$ concentrations (Figure $3 \mathrm{C}$ ) and simultaneously tracking its behavior (Figure 3E). After imaging, we segmented the fluorescence from each plane into activity units (Figure 3D) using a temporal correlation-based algorithm. ${ }^{26}$ We must note that this algorithm only utilizes correlation across time and incorporates no anatomical features, so these "activity units" may consist of individual cells, neuropil, or combinations of both. To localize segmented units within community standardized anatomical regions, we registered all imaged volumes to the reference coordinate system of the online Z-brain atlas. ${ }^{27}$

In order to identify any regions that carry information about salinity, we calculated the mutual information between each unit and the delivered salt concentration. Averaging this value across fish for each voxel of the Z-brain atlas reveals stereotypic strong $\mathrm{NaCl}$ representation within the fish's olfactory system, which includes the epithelia, bulb, and posterior telencephalon (Figure 3F). ${ }^{28}$ Of all sensory ganglia, only the rostral-most neuromasts and olfactory epithelia contain units whose activity reflects $\mathrm{NaCl}$ concentration (Figure 3G). In both of these modalities, we observe 
A

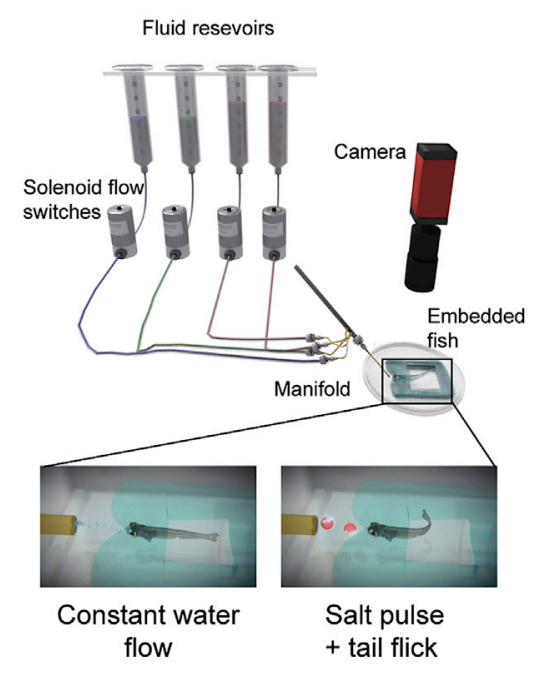

B

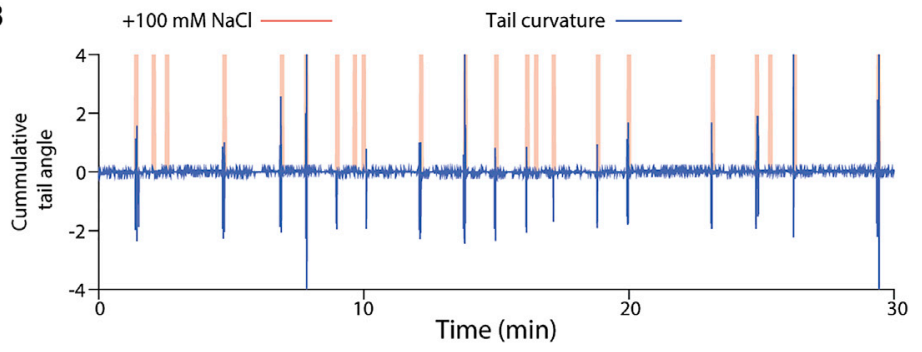

C

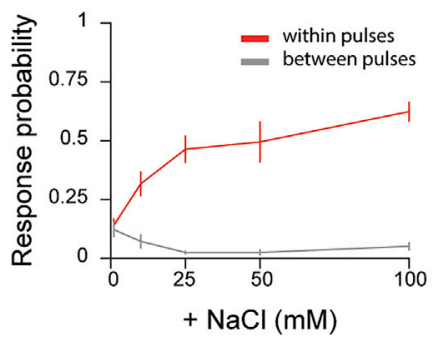

D

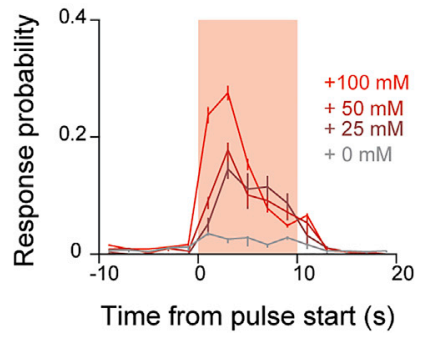

Figure 2. Head-Immobilized Larvae Respond to Salt Concentration Increases

(A) Schematic of preparation used to stimulate head-embedded larvae.

(B) Sample data from an experiment where a larva is stimulated with 10 -s pulses of $100 \mathrm{mM} \mathrm{NaCl}$ at random intervals.

(C) Probability that the larva will exhibit a behavioral response to a pulse of a given $\mathrm{NaCl}$ concentration (red) and inter-pulse spontaneous behavior rate (gray) to flowing fish water (error bars: mean \pm SEM across fish).

(D) Probability of a bout event occurring within 2-s bins for different concentrations of salt relative to the onset of a pulse (error bars: mean \pm SEM across fish). See also Figure S2.

concentration-dependent activity that, for a given concentration, was the same, on average, during trials where the animal responded and those where it did not (Figures $3 \mathrm{H}$ and 3I). Furthermore, unlike the behavior, which subsides mere seconds into a pulse, these regions sustained or even elevated their calcium levels throughout the stimulus period. This suggests that the olfactory epithelia and neuromasts of the lateral line contain sensory representations of external salt concentration and do not alone reflect when an animal will respond. To verify this, we trained support vector machines to classify either the stimulus or behavioral response from the activity of units within these regions. As predicted, these classifiers were independently able to predict the stimulus but failed to predict whether the animal produced a behavioral response (Figures $3 \mathrm{~J}$ and $3 \mathrm{~K}$ ). Thus, the neural substrate for the computations that extracts changes in salt concentration and generates behavioral responses likely occurs somewhere downstream of these primary sensory regions.

Outside of the olfactory system and neuromasts, most regions of the brain share low mutual information with the sensory stimulus, including brainstem regions previously reported to show highly stereotyped responses to tastants. ${ }^{29}$ One caveat to this observation is that our light sheet consists of a single beam that is scanned through the left eye of the animal. As such, we have likely missed salinity-related activity within the hypothalamus, as has been previously observed, ${ }^{22,30}$ or subtle activity in small nuclei proximal to the eyes (i.e., the trigeminal nucleus). Nonetheless, we do observe a midbrain cluster of units near the dorsal raphe and interpeduncular nucleus (Figure 3F), whose activity patterns share high mutual information with the animal's behavior, as well as the combination of stimulus and behavior (Figures S3A and S3B).
These areas may represent a substrate for the sensorimotor transformation of salt elevations into action, as supported by the fact that both stimulus and action can be decoded from these regions (Figures S3E and S3F). Consistent with our expectations from the behavioral dynamics, we show that, during trials where the animals respond to salt, neural activity peaks in these regions and descends to baseline within the trial (Figures S3D and S3E). However, when the animal is unresponsive, the calcium signal plateaus, suggesting that extracting changes in external salt may follow more complicated principles than a simple derivative. Future studies should be able to shed more light on these questions.

\section{Olfactory Input Is Necessary to Drive $\mathrm{NaCl}$ Avoidance Behavior}

We next wanted to define the precise roles that the olfactory system and the lateral line each play in the generation of saltavoidance behaviors. To do this, we incubated larvae in various concentrations of copper sulfate, which kills cells in both the lateral line ${ }^{31}$ and olfactory epithelia. ${ }^{32}$ After treatment and recovery periods, we examined the larvae's behavioral responses to $50 \mathrm{mM} \mathrm{NaCl}$ pulses and found their reaction rate decreased with increasing copper concentration (Figure 4A). In fact, behavioral responses to $\mathrm{NaCl}$ were essentially abolished at a copper sulfate concentration of $20 \mu \mathrm{M}$. To ensure that copper does not simply degrade all motor ability, we verified that coppertreated larvae showed no reduction in the performance of the optomotor response (Figure 4B), an innate visuo-motor behavior that leads the animal to follow whole-field motion. ${ }^{33}$

To differentiate the relative importance of olfaction and the lateral line, we examined whether the extent of damage to either 
A

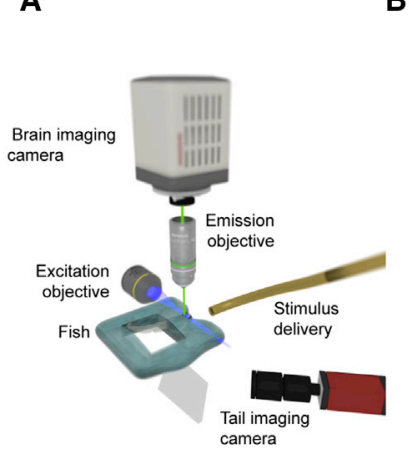

B

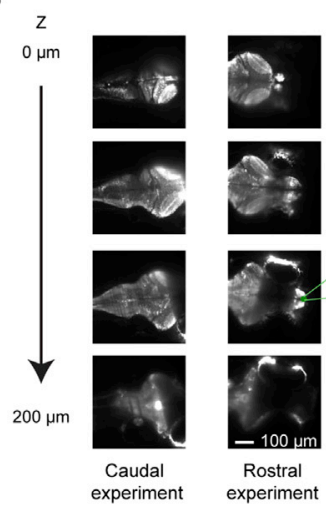

C

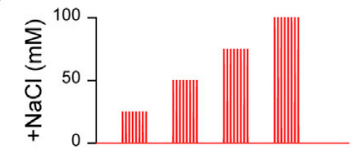

D

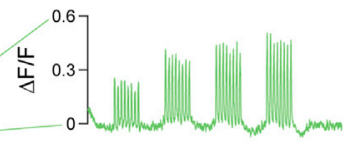

E

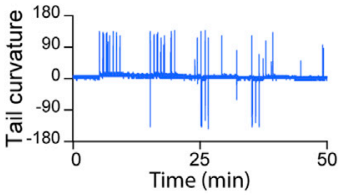

$\mathbf{F}$

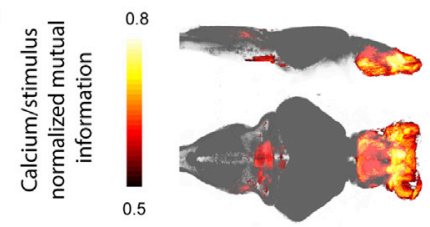

G

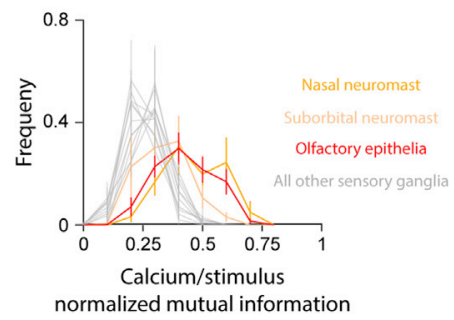

H
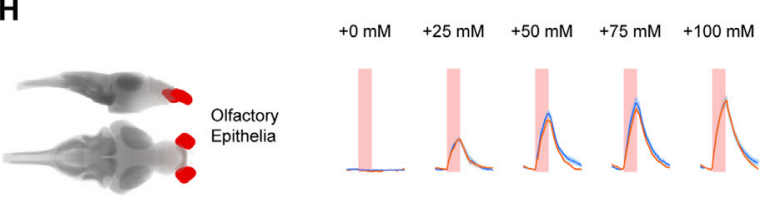

I

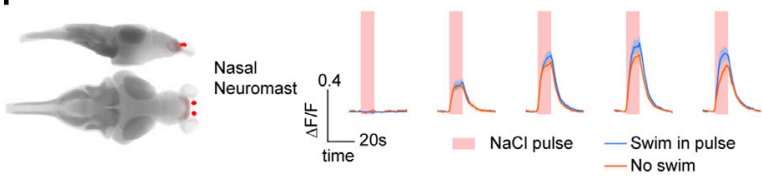

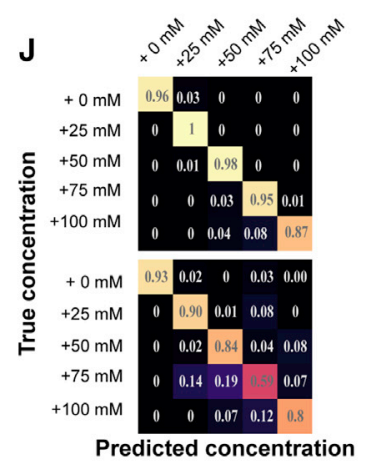

normalized mutual information

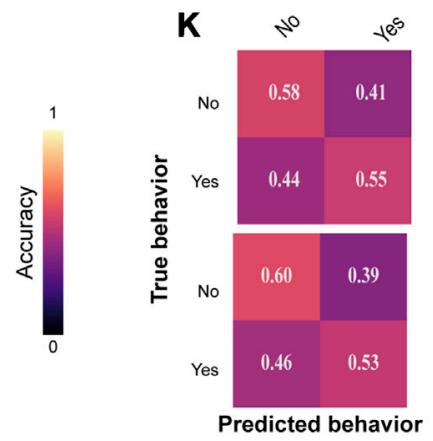

Figure 3. $\mathrm{NaCl}$ Levels Are Represented in the Olfactory System and Lateral Line

(A) Schematic of the light-sheet microscope.

(B) Sample z-slices taken within a stack of a huc:GCaMP6S transgenic larvae. Stacks were collected at $1 \mathrm{~Hz}$. Imaging experiments captured either the rostral $2 /$ $3^{\text {rds }}$ or caudal $2 / 3^{\text {rds }}$ of the fish.

(C) Arrangement of salt pulses during each experiment. Zebrafish experienced escalating concentrations of $\mathrm{NaCl}$ pulses in 5-min blocks.

(D) Example calcium signal from an activity unit in the olfactory bulb.

(E) Example tail curvature trace during imaging experiment.

(F) Average stimulus correlation at each voxel of the Z-brain across 15 fish.

(G) Histogram of normalized mutual information in units from each of the sensory ganglia in the Z-brain (error bars: mean \pm SEM across fish).

(H) Stimulus-triggered responses of top $\mathrm{NaCl}$ encoding units from the olfactory epithelia, averaged across fish, and their locations within the $Z$-brain. Responses are separated into trials where the fish swam (blue) or did not swim (purple). Mean \pm SEM across fish.

(l) Stimulus-triggered responses of top $\mathrm{NaCl}$ encoding units in the nasal neuromast averaged across fish as in $(\mathrm{H})$.

(J) Confusion matrices depicting stimulus classification accuracy of support vector machines trained from activity in the olfactory epithelia (top) and nasal neuromast (bottom).

(K) Confusion matrices depicting behavioral response classification accuracy of support vector machines trained from activity in the olfactory epithelia (top) and nasal neuromast (bottom).

See also Figure S3 and Video S1.

modality was predictive of the behavior. To quantify the remaining salt-induced calcium activity in these regions, we wished to have higher spatial resolution that was afforded by the light-sheet microscope; thus, we performed two-photon imaging to assess the extent of the removal of $\mathrm{NaCl}$ sensitivity after copper treatment (Figures 4C and 4D). We found that treatment with as little as $2 \mu \mathrm{M}$ copper sulfate already abolished all lateral line responsiveness, even though the animal continued to respond to $\mathrm{NaCl}$ (Figures 4E, S4A, and S4B). By contrast, a significant fraction of $\mathrm{NaCl}$-sensitive olfactory bulb units remained responsive at this concentration (Figures 4F and S4D). Like the behavior, these responses are only completely removed with $20 \mu \mathrm{M}$ copper sulfate, implicating the olfactory system as critical for $\mathrm{NaCl}$ avoidance.

We should note that, although the decline in $\mathrm{NaCl}$-induced behavioral response rate that results from treatment with $2 \mu \mathrm{M}$ copper sulfate is proportional to the corresponding loss of olfactory sensitivity, this does not rule out a role for the nasal neuromasts. To determine whether specific removal of the nasal neuromasts can also reduce $\mathrm{NaCl}$-triggered behaviors, we performed targeted two-photon laser ablations of both neuromasts. We found that fish that underwent this treatment, or sham ablations, did not significantly reduce their behavioral response rate, suggesting a minimal role for these neuromasts (Figures S4E and S4F). To further validate the necessity of olfaction for NaCl-triggered behaviors, we next performed a crude yet informative experiment; we rotated the fish $180^{\circ}$ relative to the stimulus. This allowed us to expose the neuromasts, as well as other assorted somatosensory systems of the tail to $\mathrm{NaCl}$, while keeping the face and the associated olfactory system largely unaffected. Under this arrangement, fish were significantly less likely to 


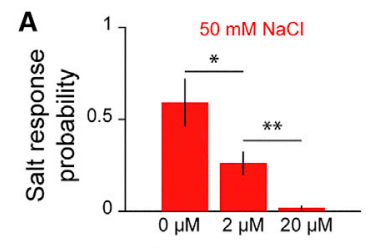

C

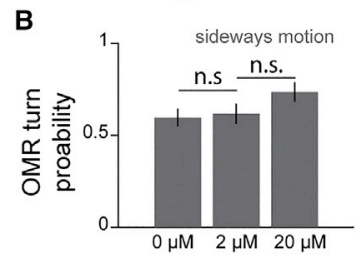

Copper treatment
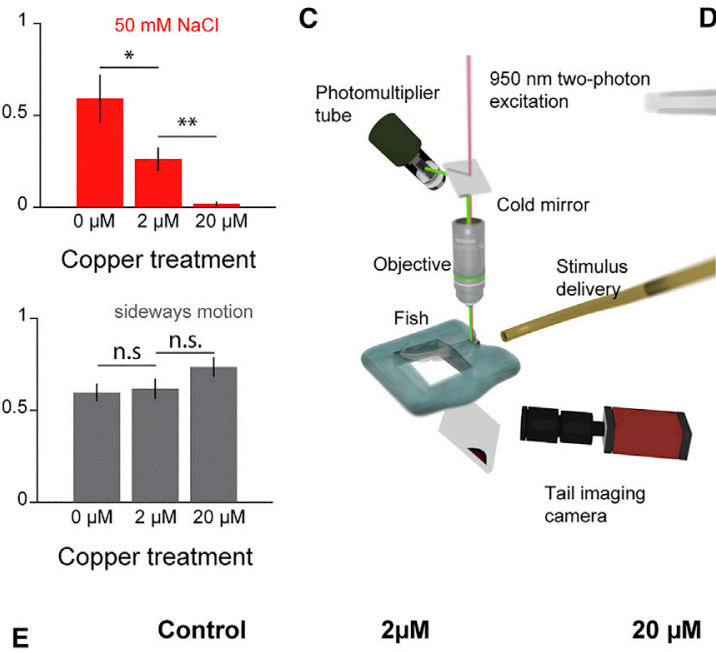

D

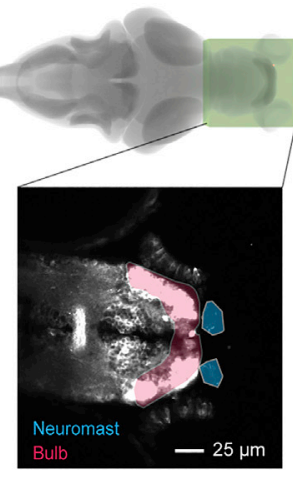

$2 \mu \mathrm{M}$

$20 \mu \mathrm{M}$
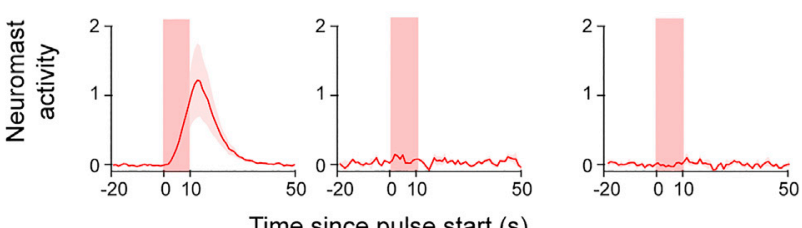

$\mathbf{F}$
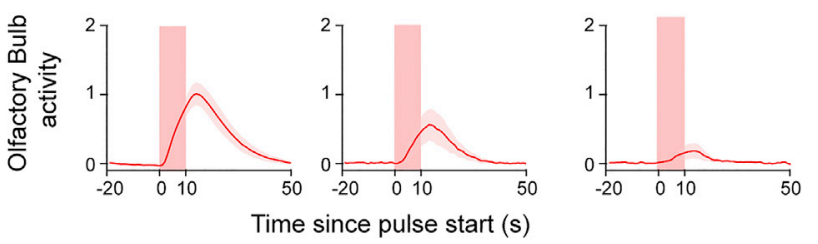

Figure 4. Salt-Avoidance Behavior Is Abolished after Removing Olfactory Sensitivity to $\mathrm{NaCl}$

(A) Behavioral response probability of tailembedded larvae to $50 \mathrm{mM} \mathrm{NaCl}$ after incubation in different concentrations of copper (two-sided t test; $p=0.034$ and $p=0.0039$; error bars: mean \pm SEM across fish; $n=6$ each).

(B) Behavioral response probability of tailembedded larvae to whole-field motion after incubation in different concentrations of copper (twosided t test; $p=0.77$ and $p=0.13$; error bars: mean \pm SEM across fish; $n=15$ each).

(C) Schematic depicting the two-photon microscope setup used to image the olfactory bulb and epithelia.

(D) Region of the brain imaged in this figure. Zoom depicts sample slices averaged over time. Shadings indicate segmented regions-neuromast (orange) and olfactory bulb (red).

(E) Stimulus-triggered averages (shading indicates SEM) of neuromast calcium activity during 50-mM $\mathrm{NaCl}$ pulses. For fish where neuromasts are fully removed, masks are drawn around where they would normally be.

(F) Stimulus-triggered averages (shading indicates SEM) of olfactory bulb calcium activity during 50$\mathrm{mM} \mathrm{NaCl}$ pulses.

See also Figure S4.

The absence of sensory neurons that detect both $\mathrm{NaCl}$ and glycine, which along with other amino acids activates members of a genetically defined subset of olfactory sensory neurons (microvillus), ${ }^{36}$ raised the

respond than when we subsequently exposed the rostrum of those same fish to $\mathrm{NaCl}$ (Figure S4G), supporting the notion that olfactory exposure is necessary to elicit $\mathrm{NaCl}$-avoidance behavior.

\section{A Sparse Subset of Olfactory Sensory Neurons Is Sensitive to $\mathrm{NaCl}$}

We next wished to determine how $\mathrm{NaCl}$ sensitivity might arise in the olfactory system. One possibility is that $\mathrm{NaCl}$ directly depolarizes the membranes of all externally contacting neurons, including all olfactory sensory cells. Based on the observations from our volumetric imaging experiments, we identified active olfactory units as those with high average correlations between trials and the trial mean $(>0.8)$ relative to shuffled traces. We found that fewer than $5 \%$ of all identified sensory units are responsive to the tested concentrations of $\mathrm{NaCl}$ (Figures $5 \mathrm{~A}-5 \mathrm{C}$ ), suggesting that $\mathrm{NaCl}$ activates a specific subpopulation of olfactory sensory neurons. To address the possibility that the large fraction of "silent" neurons may be unresponsive due to some unspecified pathological condition of our preparation, we tested whether these $\mathrm{NaCl}$-insensitive cells might respond to other odorants. To that end, we examined two compounds, one noxious to zebrafish (cadaverine) ${ }^{34}$ and the other appetitive (glycine), ${ }^{35}$ and found that both chemicals activated many $\mathrm{NaCl}$-insensitive neurons in the epithelia and bulb (Figures 5D-5I). In fact, although there is some overlap between $\mathrm{NaCl}$ and cadaverine-sensitive cells, we found zero glycine-sensitive cells in the olfactory epithelia that detect $\mathrm{NaCl}$ (Figures $5 \mathrm{H}$ and 5l). possibility that a single class of sensory neurons responds to $\mathrm{NaCl}$. However, imaging the $\mathrm{NaCl}$ response properties in two transgenic lines that label either ciliated or microvillus sensory neurons (tg(omp:Gal4/uas:GCaMP6S) ${ }^{37,38}$ for ciliated and $\operatorname{tg}$ (trpc2:Gal4/uas:GCaMP6S) ${ }^{39}$ for microvillous), revealed comparable levels of $\mathrm{NaCl}$-induced activity $(<5 \%)$ in both populations (Figure S5), thus allowing us to rule out this hypothesis.

As in mammals, the axons of zebrafish olfactory sensory neurons that express a specific receptor converge onto a restricted set of glomerular clusters in the olfactory bulb. ${ }^{40}$ To help elucidate whether $\mathrm{NaCl}$ encoding in the olfactory system follows canonical logic, we next asked whether $\mathrm{NaCl}$-sensitive sensory neurons converge onto specific glomerular clusters in the olfactory bulb or span the entire bulb. Activity detected in pan-neuronally expressed GCaMP6s may reflect secondary connections, or even bottom-up neuromodulation, and thus inflate the apparent spatial distribution within the bulb. Therefore, we chose to specifically observe whether $\mathrm{NaCl}$-sensitive olfactory sensory neurons converge onto particular locations within the bulb by imaging the axon terminals of both ciliated and microvillus sensory neurons (Figures 6A and 6B). From these experiments, we observed some activity in localized spots across most glomerular clusters as defined previously, ${ }^{41}$ but the dorsal glomeruli in the omp:gal4 fish contained the greatest, on average, amount of activity (Figure $6 \mathrm{C}$ ). This result suggests that there is chemotypic localization of $\mathrm{NaCl}$-sensitive sensory axons. To check whether the structure 
A

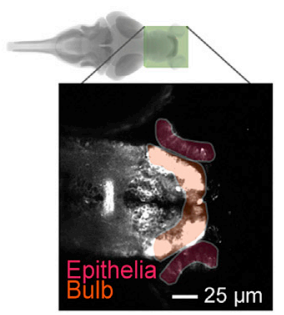

D

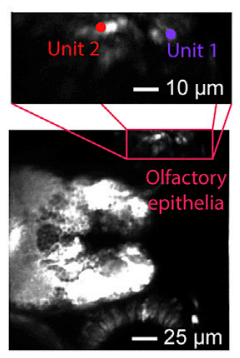

$\mathbf{F}$

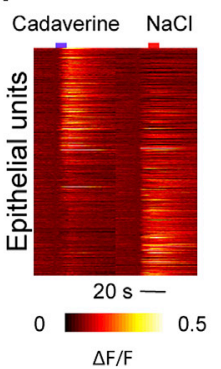

G

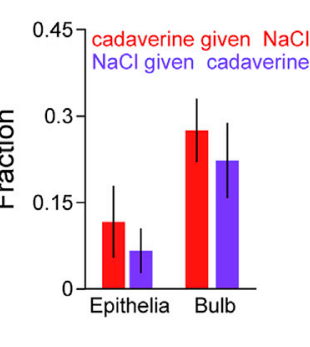

B

E
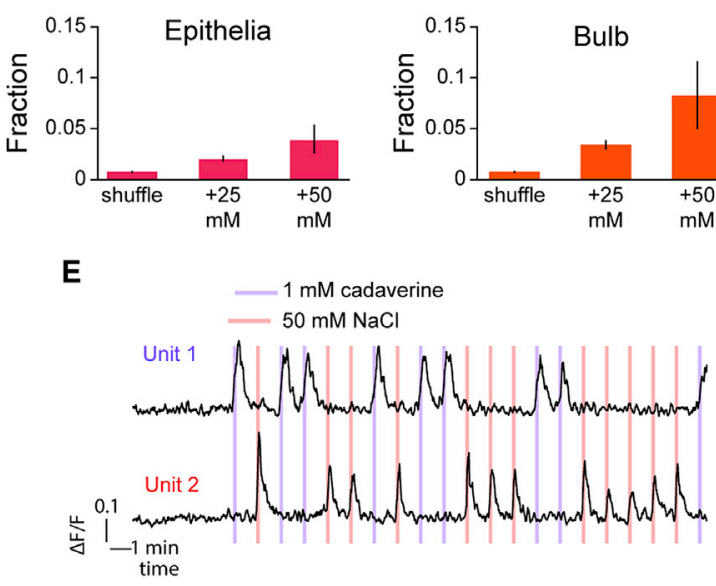

H

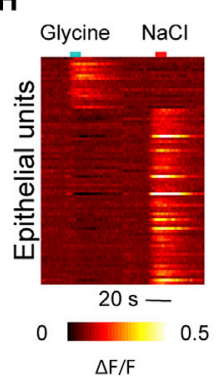

I

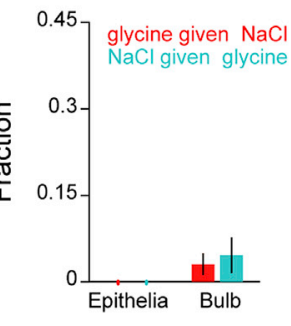

Figure 5. A Small Fraction of Functional OIfactory Sensory Neurons Responds to $\mathrm{NaCl}$ (A) Plane from the imaged region. Shadings indicate segmented regions-olfactory bulb (orange) and epithelia (red).

(B) Average fraction of active units in the olfactory epithelia of huc:GCaMP6s-positive fish during 25-mM or 50-mM pulses and after applying the same criteria to shuffled traces (error bars indicate SEM across fish; $n=3$ fish).

(C) Average fraction of active units in the olfactory bulb of huc:GCaMP6s-positive fish during $25-\mathrm{mM}$ or 50-mM pulses and after applying the same criteria to shuffled traces (error bars indicate SEM across fish; $\mathrm{n}=3$ fish).

(D) Projection across time of a sample slice imaged with $1 \mathrm{mM}$ cadaverine and $50 \mathrm{mM} \mathrm{NaCl}$. Inset depicts the location of two sample units from within the olfactory epithelia.

(E) Calcium traces of the two units depicted in $(F)$ in response to 10 -s pulses of $1 \mathrm{mM}$ cadaverine or $50 \mathrm{mM} \mathrm{NaCl}$.

(F) Heatmap depicting stimulus-triggered average activity of all responsive epithelial units to cadaverine and $\mathrm{NaCl}$.

(G) Fraction of units that are responsive to $\mathrm{NaCl}$ that are also responsive to cadaverine and vice versa (error bars indicate SEM across fish; $\mathrm{n}=3$ fish).

(H) Heatmap depicting stimulus-triggered average activity of all responsive epithelial units to glycine and $\mathrm{NaCl}$.

(I) Fraction of units that are responsive to $\mathrm{NaCl}$ that are also responsive to glycine and vice versa (error bars indicate SEM across fish; $n=3$ fish).

See also Figure 55 .

of the observed coverage is unique to $\mathrm{NaCl}$, we then compared the activity patterns of $\mathrm{NaCl}$ to those of cadaverine. In these experiments, we find that $1 \mathrm{mM}$ cadaverine and $50 \mathrm{mM}$ activate partially overlapping and similarly sized domains (Figure S6). Overall, these results are consistent with $\mathrm{NaCl}$ being detected by separate sensory neurons that express different olfactory receptors.

\section{NaCl-Sensitive Neurons Are Driven by Sodium and \\ Chloride}

Having established that sodium chloride does not directly depolarize all epithelial neurons, we next tested whether olfactory responses are driven specifically by sodium and/or chloride or whether they are tuned to an environmental shift that co-occurs with $\mathrm{NaCl}$ fluctuation (i.e., osmolarity or conductivity). To that end, we presented a given fish with random pulses of either $50 \mathrm{mM} \mathrm{NaCl}$ to identify the $\mathrm{NaCl}$-sensitive regions or a "test" chemical. Recalling the behavioral results in our gradient assay (Figure 1D), we expect that $\mathrm{NaCl}$-sensitive neurons are not osmolarity sensors, and indeed, we found that $\mathrm{NaCl}$-sensitive cells do not respond to equimolar mannitol (Figures 7A-7C). However, the larvae's behavioral avoidance of all ionic solutions (Figure 1D) offers no clues as to how the $\mathrm{NaCl}$-sensitive neurons might respond. On the one hand, these neurons might detect any change in conductivity, although on the other extreme, they could be specifically tuned to sodium or chloride. Thus, we tested a series of solutions to dissect this. When we exposed larvae to $\mathrm{KCl}$, which in effect exchanges sodium for a comparable monovalent ion, potassium, $\mathrm{NaCl}$-sensitive neurons were strongly activated (Figures $7 \mathrm{~A}-7 \mathrm{C}$ ), suggesting they are not sodium specific. To determine whether chloride drives this activity, we tested sodium conjugated with a different anion, iodide. Yet this pairing drove the population as well (Figure 7A-7C). Overall, these results suggest $\mathrm{NaCl}$-sensitive cells at least report the presence of monovalent ions. However, we found they are not conductivity sensors, as exposing the fish to isoelectric or higher concentrations of a divalent cation (magnesium chloride) generates significantly weaker, and in some cases slower, responses in these cells than to $\mathrm{NaCl}$ (Figures 7A-7C and S7A-S7D).

Our observation that fish will swim toward deionized water implies that the sensory input driving this behavior should be able to decrease from the baseline established under fish water. To test this, we examined the activity of $\mathrm{NaCl}$-sensitive olfactory neurons exposed to deionized water, and consistent with the behavior, we observed that their activity decreased (Figures S7E-S7G).

We next wished to determine how different monovalent ions influence the activity of individual $\mathrm{NaCl}$-sensitive units. When we pair chloride with cations larger or smaller than sodium, we find that a greater fraction of olfactory units responds with activity dissimilar to their $\mathrm{NaCl}$ response (Figure 7D). Pairing sodium with anions of varying size generates a similar effect, though large anions are not nearly as detrimental as large cations (compare sodium ascorbate to N-methyl-d-glucamine (NMDG) chloride; Figure 7E). This pattern suggests two possibilities: (1) there exist distinct populations of sodium-sensitive and chloride-sensitive neurons or (2) 
A $\begin{array}{cc}\text { TrpC2 } & \text { TrpC2 } \\ \text { Anatomy } & \text { Activity }(50 \mathrm{mM} \mathrm{NaCl})\end{array}$
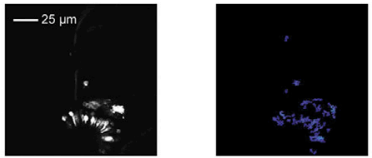

$Z=$ $-8 \mu \mathrm{m}$

B

OMP Anatomy Activity $(50 \mathrm{mM} \mathrm{NaCl})$
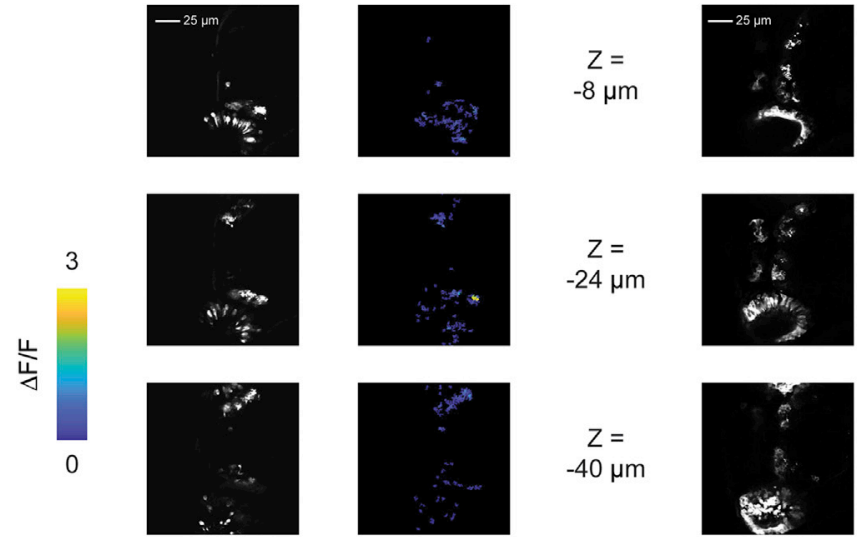

$\mathrm{Z}=$ $-40 \mu \mathrm{m}$ $Z=$
$-24 \mu m$
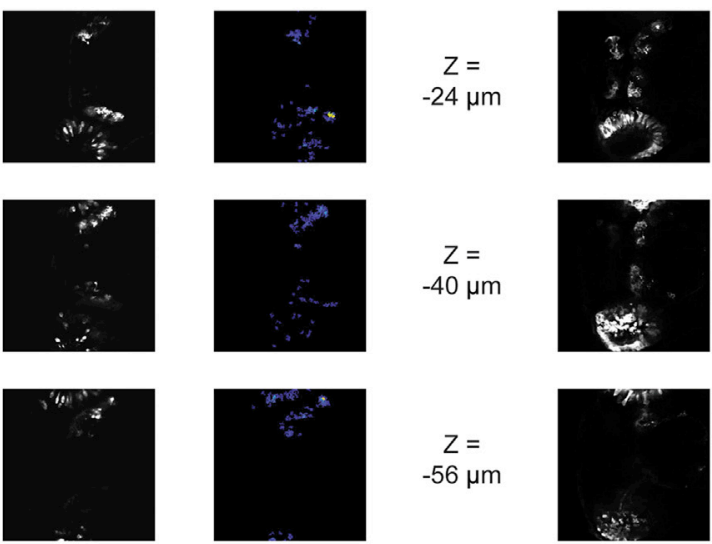

$Z=$

$-56 \mu \mathrm{m}$

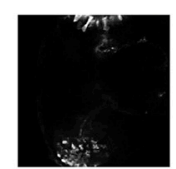

C
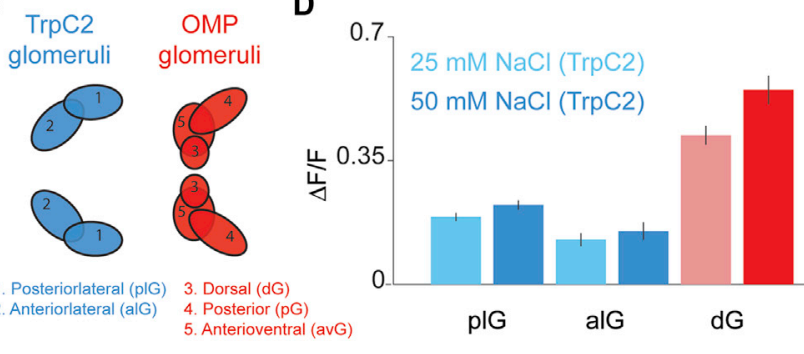

there is a single population of cells equally sensitive to both sodium and chloride (Figure 7F). To distinguish between these two hypotheses, we compared the activity in individual olfactory bulb units when triggered by adding $50 \mathrm{mM} \mathrm{RbCl}$ and $\mathrm{NaBr}$, two ion pairs that have similar effects at the population level. With these two solutions, we asked whether, when compared to stimulation with $50 \mathrm{mM} \mathrm{NaCl}$, stimulating with either rubidium (rather than sodium) or bromide (rather than chloride) modified the activity of distinct sets of neurons (Figure 7F). Although we can detect a small number of $\mathrm{NaCl}$-sensitive cells ( $\sim 7 \%$ total) that appear either sodium or chloride specific, for the vast majority of cells, we instead observe a strong correlation between the activation of each individual neuron by both $\mathrm{RbCl}$ and $\mathrm{NaBr}$ (Figure 7G-7I). Following these results, we propose that $\mathrm{NaCl}$-sensitive neurons in the zebrafish are predominantly tuned to the presence of both sodium and chloride, with perhaps a slightly greater sensitivity to sodium. To our knowledge, this dual sensitivity alludes to a novel and undescribed molecular mechanism for environmental salt detection.

\section{DISCUSSION}

Salt detection is classically considered a task for the gustatory system. This view reflects a focus on understanding salt sensing in terrestrial animals. ${ }^{5,6,42}$ On land, animals interact with environmental salt through ingestion, making taste the primary conduit
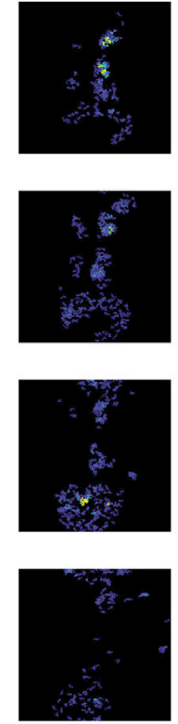

Figure 6. NaCl-Sensitive Sensory Neurons Exhibit Chemotopy in the Olfactory Bulb

(A) Sample slices from a stack taken during imaging trpc2:Gal4; uas:GCaMP6s. Left column images are maximum intensity projects, although the right column shows average maximal activity $(\Delta \mathrm{F} / \mathrm{F})$ during a $50 \mathrm{mM} \mathrm{NaCl}$ trial.

(B) Sample slices from a stack taken during imaging omp:Gal4;uas:GCaMP6s. Left column images are maximum intensity projects, although the right column shows average maximal activity during a $50 \mathrm{mM} \mathrm{NaCl}$ trial.

(C) Schematic depicting anatomical locations of glomeruli in omp and trpc2 fish used to generate (D). (D) Average across trials, cells, and fish (error bars: SEM across fish; $n=3$ ) of the maximum activity elicited by $25 \mathrm{mM} \mathrm{NaCl}$ or $50 \mathrm{mM} \mathrm{NaCl}$ within each of the glomeruli defined in (C).

See also Figure S6.

for salt-related decisions. For teleosts and other aquatic organisms, however, saltiness is a critical environmental variable, which restricts their possible habitats. Here, we have discovered that at least one species of fish incorporates olfaction to detect salt. Unlike taste, which in teleosts is reportedly exclusively used for ingestion-related decisions, ${ }^{43}$ olfaction has broad influence, ${ }^{44}$ being involved in behaviors as diverse as finding food, ${ }^{45}$ mating, ${ }^{46}$ and avoiding dangerous environments. ${ }^{34,47}$ Our finding thus suggests interesting implications for the evolution of cross-modal behaviors. Rather than endow a salt-sensitive modality, such as taste, with the ability to regulate navigational behaviors, natural selection has led to the incorporation of salt sensitivity into a different modality, olfaction, that already influences navigation across animals as diverse as flies and mice. ${ }^{48-50}$

We must acknowledge that our work only examines the zebrafish's behavioral and neural response to external salt stimuli as it exists during a brief snapshot of its development. Although our results suggest that zebrafish lack the navigational capacity until 5 days post-fertilization (Figure S1C), we cannot say whether this reflects the maturation of the sensory tissue or behavioral capabilities. An earlier study that used acute olfactory stimuli ${ }^{51}$ found some ability of younger larvae (3 days post fertilization [dpf]) to avoid noxious odorants, suggesting the sensory capacity may already exist. Furthermore, as the animal matures beyond $7 \mathrm{dpf}$, it may incorporate additional sensory modalities. For example, the sensory epithelia in the gills are an intriguing candidate, given that they are the major site for ionic regulation in the adult fish ${ }^{52}$ and play a key sensory role for detecting hypoxia after $7 \mathrm{dpf}^{13}$

When we investigated the detailed dynamics of the salt gradient navigation, we found that larval zebrafish modify their behavior during the onset of increased salinity (Figures $1 \mathrm{~F}$ and 2D). Yet the heightened activity in the olfactory system is sustained throughout the duration of elevated salt. This raises several questions. First, how does the larva's brain generate 
A

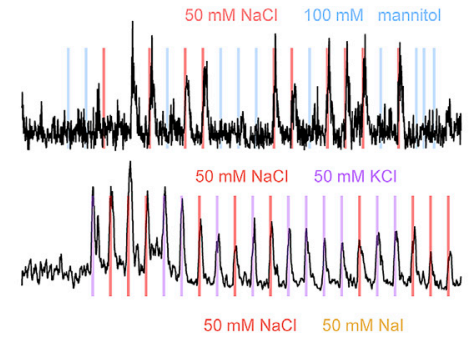

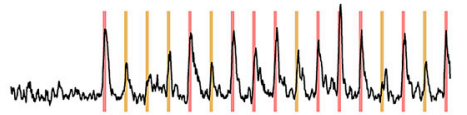

$50 \mathrm{mM} \mathrm{NaCl} \quad 25 \mathrm{mM} \mathrm{MgCl} 2$

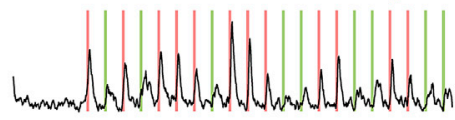
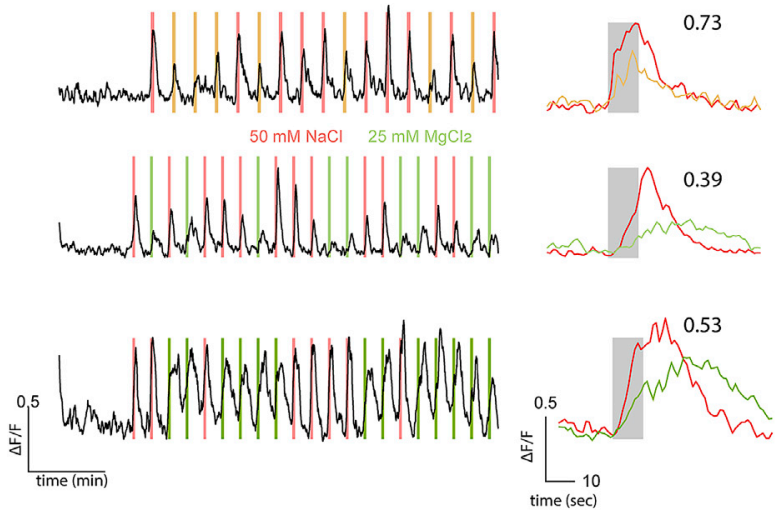

B $\quad 0.03$
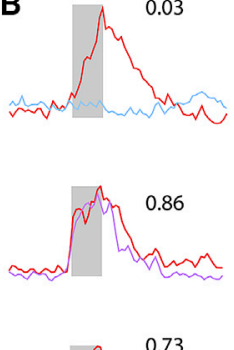

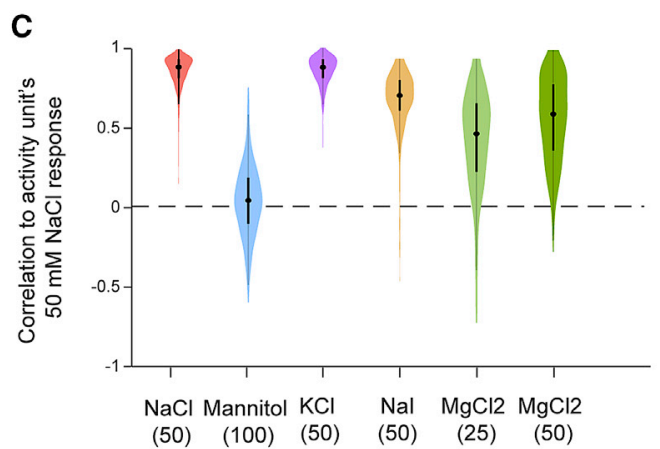

E
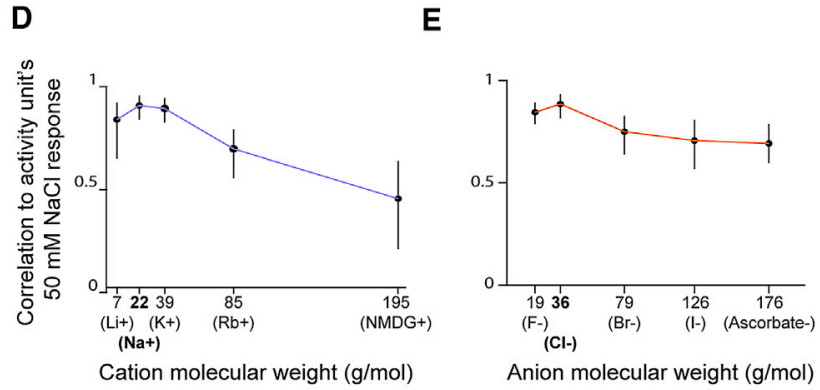

$\mathbf{F}$
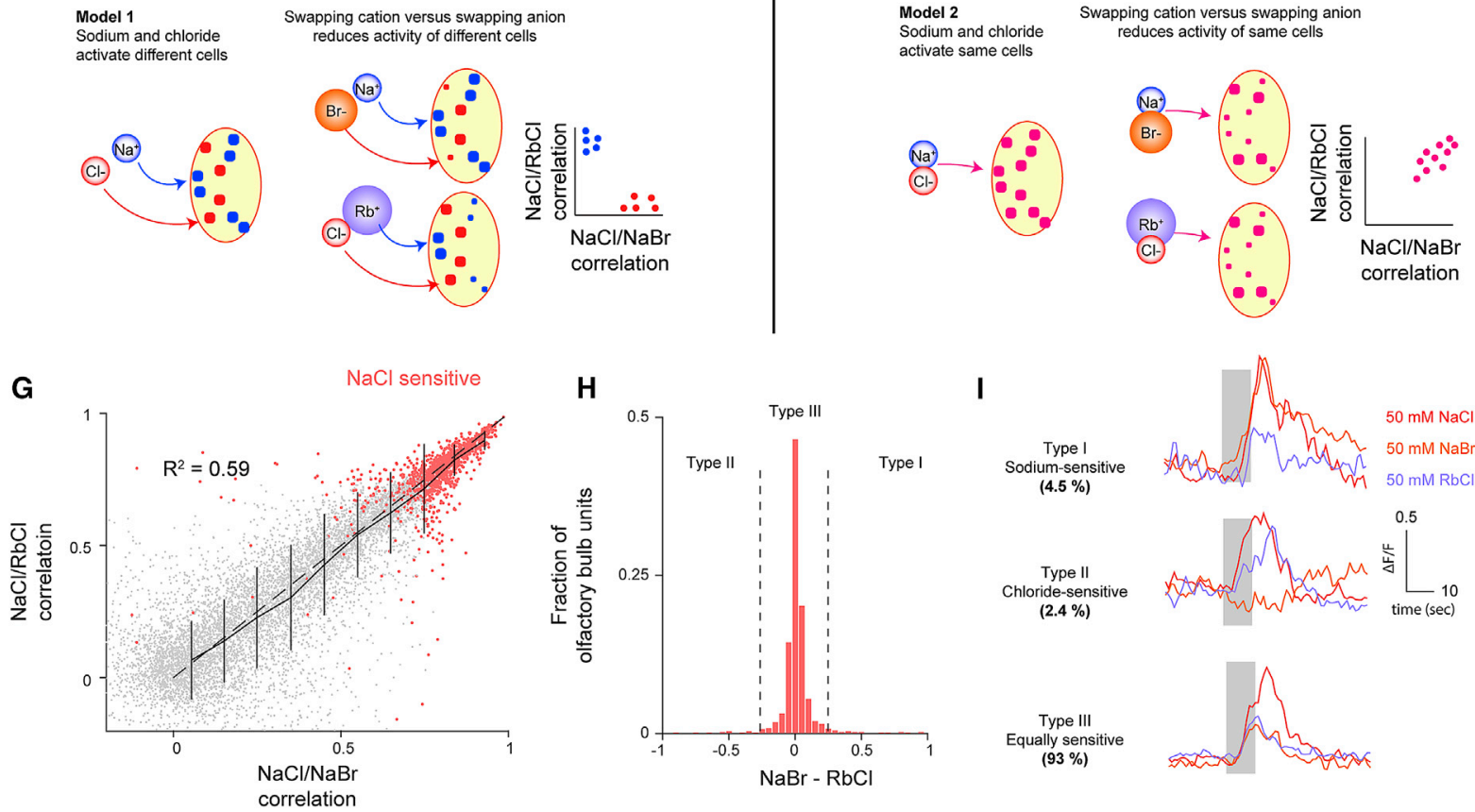

I
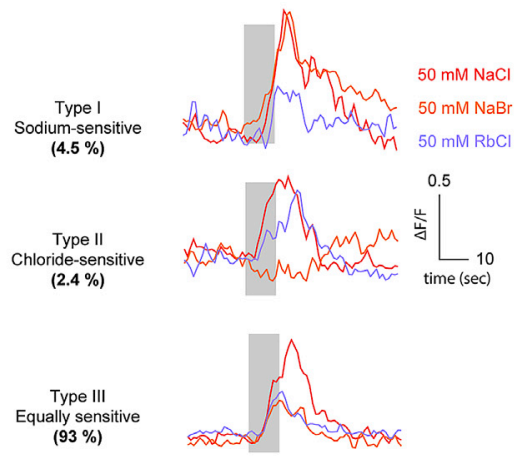

Figure 7. NaCl-Sensitive Olfactory Bulb Units Are Sensitive to Sodium and Chloride lons

(A) Sample activity trace from an olfactory bulb unit while the fish is stimulated with pulses of $50 \mathrm{mM} \mathrm{NaCl}$ and a test chemical.

(B) Stimulus-triggered averages of the activity unit in (A) in response to $50 \mathrm{mM} \mathrm{NaCl}$ and a test chemical. For each unit examined, the correlation between the $\mathrm{NaCl}$-triggered response and test-triggered response is reported (i.e., 0.03 for the sample mannitol unit). These values correspond roughly to the medians of the distributions in (C).

(C) Distribution of the correlation of each olfactory bulb activity unit's $\mathrm{NaCl}$ response to different chemicals. Bars in violin plots indicate median $\pm 25 \%$.

(D) Median (error bars $25 \%-75 \%$ ) correlation of $\mathrm{NaCl}$ activity with different cation/chloride combinations.

(E) Median (error bars $25 \%-75 \%$ ) correlation of $\mathrm{NaCl}$ activity with different anion/sodium combinations.

(F) Cartoon depicting the two models of $\mathrm{NaCl}$ sensitivity and expected results from stimulating $\mathrm{NaCl}$-sensitive cells with $\mathrm{NaBr}$ and $\mathrm{RbCl}$ given each model.

(G) Scatterplot of correlation between $\mathrm{NaCl}$ and $\mathrm{RbCl}$ responses versus correlation between $\mathrm{NaCl}$ and $\mathrm{NaBr}$ responses for each activity unit tested (indicated by gray dots; $n=3$ fish). R-squared calculated from the correlation of all individual units from within the three olfactory bulbs. Solid line indicates average and 
the relevant derivative? At present, the nature of this computation is unclear, and we may be missing relevant signals from, for instance, the hypothalamus. Although the interpeduncular nucleus as well as the raphe seem like potential sites to explore this further, their activity profiles do not reflect a pure derivative of salt concentration. In particular, the lack of neural adaptation during trials without a behavioral response obfuscates the possible neural implementation. Future studies should further dissect the nature of the behavioral algorithm by which changes in salt concentration are detected, as this may offer clear constraints to the neural mechanisms. For example, feedback from an efferent relay of the animal's motor activity may modulate how the animal's brain processes sustained salt elevation.

Second, why does the fish expend extra energy to sustain high firing rates in the olfactory system when the behaviorally relevant information only lasts a few seconds? Even the baseline activity in normal fish water is well above the minimal activity during exposure to deionized water. In line with a hypothesis discussed in Lovett-Barron et al., ${ }^{22}$ retention of information about the surrounding salinity might be necessary for regulating non-motor-related functions. After early attempts to escape salt fail, the animal may still survive if it can hormonally regulate its ion balance. For example, salt information from the olfactory system may directly regulate hypothalamic release of prolactin $^{53}$ or cortisol $^{54}$ to balance ion uptake and excretion, respectively.

At present, the molecular mechanisms that endow hair cells and some olfactory sensory neurons with $\mathrm{NaCl}$ sensitivity are unclear. It is possible that salt is detected entirely through combinatorial activity of different olfactory receptors, similar to most classical odorants. Alternatively, there may be a single receptor that is expressed at varying levels across the olfactory system. The coactivity of the lateral line suggests the latter scenario may at least play a partial role, though we can only speculate about that receptor's identity. Here, we observe that zebrafish larvae avoid levels of salt concentrations that fall within the operating range of the epithelial sodium channels that are essential for detecting appetitive salt in land animals. ${ }^{5}$ Yet fish lack these channels, suggesting they would need to utilize some other mechanism. Indeed, the response profile of the larvae's $\mathrm{NaCl}$-sensitive olfactory neurons suggests a different mechanism. Instead of being specifically activated by sodium like the terrestrial sodium channels, these cells are broadly activated by both monovalent cations and anions. This response profile is also distinct from known molecules responsible for detecting internal sodium, which are either specific to high (>100 mM) concentrations of sodium ${ }^{55}$ or are general osmolarity sensors. ${ }^{56}$ Whether the cellular responses result from the action of multiple receptors and channels or from a single protein, i.e., an ion-pair receptor, ${ }^{57}$ is unclear.

To our knowledge, the receptor that most closely matches the ion-sensitivity profile of the larvae's olfactory sensory neurons is pickpocket23 in the fruit fly. ${ }^{58}$ Like the zebrafish cells, this protein is broadly sensitive to monovalent ions and not osmolarity. However, the relative influence of the cation and anion is undescribed. Further, much higher concentrations $(>200 \mathrm{mM})$ are needed to drive activity in pickpocket23-positive cells than in the zebrafish sensory neurons, suggesting, at a minimum, that zebrafish use alternative secondary mechanisms. Identifying the responsible molecules should be the work of future studies. The robust nature of the avoidance behavior, as well as the ability to test many larvae simultaneously in our lane assay, make this question well suited for a broad genetic screen.

\section{STAR $\star$ METHODS}

Detailed methods are provided in the online version of this paper and include the following:

- KEY RESOURCES TABLE

- RESOURCE AVAILABILITY

O Lead contact

O Materials availability

Data and code availability

- EXPERIMENTAL MODEL AND SUBJECT DETAILS

- METHOD DETAILS

○ Free-swimming place-preference assay

- Head-embedded chemical stimulation

O Chemical treatments

○ Light-sheet microscopy

O Two-photon microscopy and ablations

- QUANTIFICATION AND STATISTICAL ANALYSIS

O Behavior analyses

Free swimming simulations

Image Analysis

\section{SUPPLEMENTAL INFORMATION}

Supplemental Information can be found online at https://doi.org/10.1016/j. cub.2020.11.051.

\section{ACKNOWLEDGMENTS}

K.J.H. received funding from the Harvard Minds Brains and Behavior Initiative. F.E. received funding from the National Institutes of Health (U19NS104653, R43OD024879, and 2R44OD024879), the National Science Foundation (IIS1912293), and the Simons Foundation (SCGB 542973). We thank all members of the Engert lab for support and advice throughout the project. We also thank Mariela Petkova and Hanna Zwaka for providing valuable feedback and suggestions to improve the manuscript.

\section{AUTHOR CONTRIBUTIONS}

K.J.H. conceived the project with F.E. and performed all experiments. D.G.-N. and T.P. built the light-sheet and two-photon microscopes. K.J.H. analyzed the data. K.J.H. wrote the manuscript with input from F.E. and D.G.-N.

variance of $\mathrm{NaCl} / \mathrm{RbCl}$ correlation for binned $\mathrm{NaCl} / \mathrm{NaBr}$ correlations. Error bars indicate variance of $\mathrm{RbCl}$ correlations for a given $\mathrm{NaBr}$ bin. Dashed line indicates unity. Red dots indicate units judged as $\mathrm{NaCl}$ sensitive (inter-trial correlation $>0.8$ ).

(H) Histogram of $\mathrm{NaCl}$-sensitive cells in (G) of the difference between $\mathrm{NaCl} / \mathrm{NaBr}$ correlation and $\mathrm{NaCl} / \mathrm{RbCl}$ correlation. Dashed lines indicate cutoff separating chloride sensitive $(<-0.25)$, equal sensitive, and sodium sensitive $(>0.25)$.

(l) Sample stimulus-triggered averages of cells from each type for $\mathrm{NaCl}, \mathrm{NaBr}$, and $\mathrm{RbCl}$.

See also Figure S7. 


\section{DECLARATION OF INTERESTS}

The authors declare no competing interests.

Received: September 14, 2020

Revised: November 17, 2020

Accepted: November 18, 2020

Published: December 17, 2020

\section{REFERENCES}

1. Matsuda, T., Hiyama, T.Y., Niimura, F., Matsusaka, T., Fukamizu, A., Kobayashi, K., Kobayashi, K., and Noda, M. (2017). Distinct neural mechanisms for the control of thirst and salt appetite in the subfornical organ. Nat. Neurosci. 20, 230-241.

2. Buggy, J., and Fisher, A.E. (1974). Evidence for a dual central role for angiotensin in water and sodium intake. Nature 250, 733-735.

3. Keys, A., and Willmer, E.N. (1932). "Chloride secreting cells" in the gills of fishes, with special reference to the common eel. J. Physiol. 76, 368-378.2.

4. Karnaky, K.J., Jr., Degnan, K.J., and Zadunaisky, J.A. (1977). Chloride transport across isolated opercular epithelium of killifish: a membrane rich in chloride cells. Science 195, 203-205.

5. Chandrashekar, J., Kuhn, C., Oka, Y., Yarmolinsky, D.A., Hummler, E., Ryba, N.J.P., and Zuker, C.S. (2010). The cells and peripheral representation of sodium taste in mice. Nature 464, 297-301.

6. Oka, Y., Butnaru, M., von Buchholtz, L., Ryba, N.J.P., and Zuker, C.S. (2013). High salt recruits aversive taste pathways. Nature 494, 472-475.

7. Zhang, Y.V., Ni, J., and Montell, C. (2013). The molecular basis for attractive salt-taste coding in Drosophila. Science 340, 1334-1338.

8. Lee, M.J., Sung, H.Y., Jo, H., Kim, H.-W., Choi, M.S., Kwon, J.Y., and Kang, K. (2017). lonotropic receptor $76 \mathrm{~b}$ is required for gustatory aversion to excessive $\mathrm{Na}+$ in Drosophila. Mol. Cells 40, 787-795.

9. Nomura, K., Nakanishi, M., Ishidate, F., Iwata, K., and Taruno, A. (2020). All-electrical $\mathrm{Ca}^{2+}$-independent signal transduction mediates attractive sodium taste in taste buds. Neuron 106, 816-829.e6.

10. Hubbard, P.C., Ingleton, P.M., Bendell, L.A., Barata, E.N., and Canário, A.V.M. (2002). Olfactory sensitivity to changes in environmental [Ca(2+)] in the freshwater teleost Carassius auratus: an olfactory role for the $\mathrm{Ca}(2+)$-sensing receptor? J. Exp. Biol. 205, 2755-2764.

11. Koide, T., Yabuki, Y., and Yoshihara, Y. (2018). Terminal nerve GnRH3 neurons mediate slow avoidance of carbon dioxide in larval zebrafish. Cell Rep. 22, 1115-1123.

12. Kotrschal, K., Krautgartner, W.D., and Hansen, A. (1997). Ontogeny of the solitary chemosensory cells in the zebrafish, Danio rerio. Chem. Senses $22,111-118$

13. Jonz, M.G., and Nurse, C.A. (2005). Development of oxygen sensing in the gills of zebrafish. J. Exp. Biol. 208, 1537-1549.

14. Katsuki, Y., Hashimoto, T., and Kendall, J.I. (1971). The chemoreception in the lateral-line organs of teleosts. Jpn. J. Physiol. 21, 99-118.

15. Venkatesh, B., Kirkness, E.F., Loh, Y.-H., Halpern, A.L., Lee, A.P., Johnson, J., Dandona, N., Viswanathan, L.D., Tay, A., Venter, J.C., et al. (2007). Survey sequencing and comparative analysis of the elephant shark (Callorhinchus milii) genome. PLoS Biol. 5, e101.

16. Oike, H., Nagai, T., Furuyama, A., Okada, S., Aihara, Y., Ishimaru, Y., Marui, T., Matsumoto, I., Misaka, T., and Abe, K. (2007). Characterization of ligands for fish taste receptors. J. Neurosci. 27, 5584-5592.

17. Spence, R., Fatema, M.K., Reichard, M., Huq, K.A., Wahab, M.A., Ahmed, Z.F., and Smith, C. (2006). The distribution and habitat preferences of the zebrafish in Bangladesh. J. Fish Biol. 69, 1435-1448.

18. Spence, R., Gerlach, G., Lawrence, C., and Smith, C. (2008). The behaviour and ecology of the zebrafish, Danio rerio. Biol. Rev. Camb. Philos. Soc. 83, 13-34.

19. Salehin, M., Chowdhury, M.M.A., Clarke, D., Mondal, S., Nowreen, S., Jahiruddin, M., and Haque, A. (2018). Mechanisms and drivers of soil salinity in coastal Bangladesh. In Ecosystem Services for Well-Being in Deltas: Integrated Assessment for Policy Analysis, R.J. Nicholls, C.W. Hutton, W.N. Adger, S.E. Hanson, M.M. Rahman, and M. Salehin, eds. (Springer International Publishing), pp. 333-347.

20. Yeh, C.-M., Glöck, M., and Ryu, S. (2013). An optimized whole-body cortisol quantification method for assessing stress levels in larval zebrafish. PLoS ONE 8, e79406.

21. Ryu, S., and De Marco, R.J. (2017). Performance on innate behaviour during early development as a function of stress level. Sci. Rep. 7, 7840.

22. Lovett-Barron, M., Chen, R., Bradbury, S., Andalman, A.S., Wagle, M., Guo, S., and Deisseroth, K. (2020). Multiple convergent hypothalamusbrainstem circuits drive defensive behavior. Nat. Neurosci. 23, 959-967.

23. Block, S.M., Segall, J.E., and Berg, H.C. (1982). Impulse responses in bacterial chemotaxis. Cell 31, 215-226.

24. Haesemeyer, M., Robson, D.N., Li, J.M., Schier, A.F., and Engert, F. (2015). The structure and timescales of heat perception in larval zebrafish. Cell Syst. 1, 338-348.

25. Kim, D.H., Kim, J., Marques, J.C., Grama, A., Hildebrand, D.G.C., Gu, W., Li, J.M., and Robson, D.N. (2017). Pan-neuronal calcium imaging with cellular resolution in freely swimming zebrafish. Nat. Methods 14, 1107-1114.

26. Portugues, R., Feierstein, C.E., Engert, F., and Orger, M.B. (2014). Wholebrain activity maps reveal stereotyped, distributed networks for visuomotor behavior. Neuron 81, 1328-1343.

27. Randlett, O., Wee, C.L., Naumann, E.A., Nnaemeka, O., Schoppik, D., Fitzgerald, J.E., Portugues, R., Lacoste, A.M.B., Riegler, C., Engert, F., and Schier, A.F. (2015). Whole-brain activity mapping onto a zebrafish brain atlas. Nat. Methods 12, 1039-1046.

28. Yaksi, E., von Saint Paul, F., Niessing, J., Bundschuh, S.T., and Friedrich, R.W. (2009). Transformation of odor representations in target areas of the olfactory bulb. Nat. Neurosci. 12, 474-482.

29. Vendrell-Llopis, N., and Yaksi, E. (2015). Evolutionary conserved brainstem circuits encode category, concentration and mixtures of taste. Sci. Rep. 5, 17825

30. Vom Berg-Maurer, C.M., Trivedi, C.A., Bollmann, J.H., De Marco, R.J., and Ryu, S. (2016). The severity of acute stress is represented by increased synchronous activity and recruitment of hypothalamic $\mathrm{CRH}$ neurons. J. Neurosci. 36, 3350-3362.

31. Hernández, P.P., Moreno, V., Olivari, F.A., and Allende, M.L. (2006). Sublethal concentrations of waterborne copper are toxic to lateral line neuromasts in zebrafish (Danio rerio). Hear. Res. 213, 1-10.

32. Ma, E.Y., Heffern, K., Cheresh, J., and Gallagher, E.P. (2018). Differentia copper-induced death and regeneration of olfactory sensory neuron populations and neurobehavioral function in larval zebrafish. Neurotoxicology $69,141-151$.

33. Orger, M.B., Smear, M.C., Anstis, S.M., and Baier, H. (2000). Perception of Fourier and non-Fourier motion by larval zebrafish. Nat. Neurosci. 3, 1128-1133.

34. Hussain, A., Saraiva, L.R., Ferrero, D.M., Ahuja, G., Krishna, V.S., Liberles, S.D., and Korsching, S.I. (2013). High-affinity olfactory receptor for the death-associated odor cadaverine. Proc. Natl. Acad. Sci. USA 110, 19579-19584.

35. Lindsay, S.M., and Vogt, R.G. (2004). Behavioral responses of newly hatched zebrafish (Danio rerio) to amino acid chemostimulants. Chem. Senses 29, 93-100.

36. Koide, T., Miyasaka, N., Morimoto, K., Asakawa, K., Urasaki, A., Kawakami, K., and Yoshihara, Y. (2009). Olfactory neural circuitry for attraction to amino acids revealed by transposon-mediated gene trap approach in zebrafish. Proc. Natl. Acad. Sci. USA 106, 9884-9889.

37. Lakhina, V., Marcaccio, C.L., Shao, X., Lush, M.E., Jain, R.A., Fujimoto, E., Bonkowsky, J.L., Granato, M., and Raper, J.A. (2012). Netrin/DCC signaling guides olfactory sensory axons to their correct location in the olfactory bulb. J. Neurosci. 32, 4440-4456.

38. Muto, A., Lal, P., Ailani, D., Abe, G., Itoh, M., and Kawakami, K. (2017) Activation of the hypothalamic feeding centre upon visual prey detection. Nat. Commun. 8, 15029. 
39. DeMaria, S., Berke, A.P., Van Name, E., Heravian, A., Ferreira, T., and Ngai, J. (2013). Role of a ubiquitously expressed receptor in the vertebrate olfactory system. J. Neurosci. 33, 15235-15247.

40. Dang, P., Fisher, S.A., Stefanik, D.J., Kim, J., and Raper, J.A. (2018). Coordination of olfactory receptor choice with guidance receptor expression and function in olfactory sensory neurons. PLoS Genet. 14, e1007164.

41. Miyasaka, N., Morimoto, K., Tsubokawa, T., Higashijima, S., Okamoto, H., and Yoshihara, Y. (2009). From the olfactory bulb to higher brain centers: genetic visualization of secondary olfactory pathways in zebrafish. J. Neurosci. 29, 4756-4767.

42. Wang, Z., Singhvi, A., Kong, P., and Scott, K. (2004). Taste representations in the Drosophila brain. Cell 117, 981-991.

43. Morais, S. (2017). The physiology of taste in fish: potential implications for feeding stimulation and gut chemical sensing. Rev. Fish. Sci. Aquacult. 25, 133-149.

44. Kermen, F., Darnet, L., Wiest, C., Palumbo, F., Bechert, J., Uslu, O., and Yaksi, E. (2020). Stimulus-specific behavioral responses of zebrafish to a large range of odors exhibit individual variability. BMC Biol. 18, 66.

45. Wakisaka, N., Miyasaka, N., Koide, T., Masuda, M., Hiraki-Kajiyama, T., and Yoshihara, Y. (2017). An adenosine receptor for olfaction in fish Curr. Biol. 27, 1437-1447.e4.

46. Yabuki, Y., Koide, T., Miyasaka, N., Wakisaka, N., Masuda, M., Ohkura, M., Nakai, J., Tsuge, K., Tsuchiya, S., Sugimoto, Y., and Yoshihara, Y (2016). Olfactory receptor for prostaglandin F2 $\alpha$ mediates male fish courtship behavior. Nat. Neurosci. 19, 897-904.

47. Mathuru, A.S., Kibat, C., Cheong, W.F., Shui, G., Wenk, M.R., Friedrich, R.W., and Jesuthasan, S. (2012). Chondroitin fragments are odorants that trigger fear behavior in fish. Curr. Biol. 22, 538-544.

48. Gire, D.H., Kapoor, V., Arrighi-Allisan, A., Seminara, A., and Murthy, V.N. (2016). Mice develop efficient strategies for foraging and navigation using complex natural stimuli. Curr. Biol. 26, 1261-1273.

49. Baker, K.L., Dickinson, M., Findley, T.M., Gire, D.H., Louis, M., Suver, M.P., Verhagen, J.V., Nagel, K.I., and Smear, M.C. (2018). Algorithms for olfactory search across species. J. Neurosci. 38, 9383-9389.
50. Draft, R.W., McGill, M.R., Kapoor, V., and Murthy, V.N. (2018). Carpenter ants use diverse antennae sampling strategies to track odor trails. J. Exp. Biol. 221, jeb185124.

51. Vitebsky, A., Reyes, R., Sanderson, M.J., Michel, W.C., and Whitlock, K.E. (2005). Isolation and characterization of the laure olfactory behavioral mutant in the zebrafish, Danio rerio. Dev. Dyn. 234, 229-242.

52. Hwang, P.-P., and Chou, M.-Y. (2013). Zebrafish as an animal model to study ion homeostasis. Pflugers Arch. 465, 1233-1247.

53. Breves, J.P., McCormick, S.D., and Karlstrom, R.O. (2014). Prolactin and teleost ionocytes: new insights into cellular and molecular targets of prolactin in vertebrate epithelia. Gen. Comp. Endocrinol. 203, 21-28.

54. Kumai, Y., Bernier, N.J., and Perry, S.F. (2014). Angiotensin-II promotes $\mathrm{Na}+$ uptake in larval zebrafish, Danio rerio, in acidic and ion-poor water. J. Endocrinol. 220, 195-205.

55. Hiyama, T.Y., Watanabe, E., Okado, H., and Noda, M. (2004). The subfornical organ is the primary locus of sodium-level sensing by $\mathrm{Na}(\mathrm{x})$ sodium channels for the control of salt-intake behavior. J. Neurosci. 24, 9276-9281.

56. Blackburn, R.E., Samson, W.K., Fulton, R.J., Stricker, E.M., and Verbalis, J.G. (1993). Central oxytocin inhibition of salt appetite in rats: evidence for differential sensing of plasma sodium and osmolality. Proc. Natl. Acad. Sci. USA 90, 10380-10384.

57. Kim, S.K., and Sessler, J.L. (2010). Ion pair receptors. Chem. Soc. Rev. 39 3784-3809.

58. Jaeger, A.H., Stanley, M., Weiss, Z.F., Musso, P.-Y., Chan, R.C., Zhang, H., Feldman-Kiss, D., and Gordon, M.D. (2018). A complex peripheral code for salt taste in Drosophila. eLife 7, e37167.

59. Panier, T., Romano, S.A., Olive, R., Pietri, T., Sumbre, G., Candelier, R., and Debrégeas, G. (2013). Fast functional imaging of multiple brain regions in intact zebrafish larvae using selective plane illumination microscopy. Front. Neural Circuits 7, 65.

60. Huang, K.-H., Ahrens, M.B., Dunn, T.W., and Engert, F. (2013). Spinal projection neurons control turning behaviors in zebrafish. Curr. Biol. 23, 1566-1573.

61. Rohlfing, T., and Maurer, C.R., Jr. (2003). Nonrigid image registration in shared-memory multiprocessor environments with application to brains, breasts, and bees. IEEE Trans. Inf. Technol. Biomed. 7, 16-25. 


\section{STAR $\star$ METHODS}

\section{KEY RESOURCES TABLE}

\begin{tabular}{|c|c|c|}
\hline REAGENT or RESOURCE & SOURCE & IDENTIFIER \\
\hline \multicolumn{3}{|l|}{ Chemicals, Peptides, and Recombinant Proteins } \\
\hline Cadaverine & Sigma aldrich & D22606-5G \\
\hline Low melting point agarose & Invitrogen & $16520-100$ \\
\hline \multicolumn{3}{|l|}{ Experimental Models: Organisms/Strains } \\
\hline 4-7 days post fertilization; Danio rerio: Tg(elavl3:GCaMP6s; nacre +/- or -/-) & 25 & a13203 \\
\hline 6-7 days post fertilization; Danio rerio: Tg(omp:Gal4;uas:GCaMP6s; nacre -/-) & 37,38 & N/A \\
\hline 6-7 days post fertilization; Danio rerio: Tg(trpc2:Gal4; uas:GCaMP6s) & 38,39 & N/A \\
\hline \multicolumn{3}{|l|}{ Software and Algorithms } \\
\hline LabVIEW 2017 software (running microscopes and behavioral rigs) & This study & $\mathrm{N} / \mathrm{A}$ \\
\hline MATLAB 2017b analysis software & This study & $\begin{array}{l}\text { https://github.com/kjherrera23/ } \\
\text { SaltSensing }\end{array}$ \\
\hline
\end{tabular}

\section{RESOURCE AVAILABILITY}

\section{Lead contact}

Further information and questions or inquiries about data and resources should be directed to and will be fulfilled by the lead author, Kristian J Herrera (kjherrera23@gmail.com).

Materials availability

No unique reagents were created for this study.

Data and code availability

Code used for data analysis and simulations is available at https://github.com/kjherrera23/SaltSensing. Due to their large size, the datasets were not made available on a public repository, but are available from the lead author upon request.

\section{EXPERIMENTAL MODEL AND SUBJECT DETAILS}

Unless otherwise noted, all fish used were the offspring of crosses of Tg(huc:GCaMP6s) positive and nacre \pm parents ${ }^{25}$. Olfactory sensory neuron imaging experiments were performed using the offspring of parents that were Tg(uas:GCaMP6S) ${ }^{38}$ and either Tg(omp:Gal4) ${ }^{37}$ or $\operatorname{Tg}\left(\right.$ trpc2:Gal4) ${ }^{39}$. Embryos were raised at 27 degrees celsius. For the first 24 hours embryos developed in embryo water plus methylene blue. Afterward, larvae were exclusively raised in filtered (200 nm pore size) facility water. Water was exchanged every day. The larvae were fed live paramecia starting at 4 days post-fertilization. Experiments were performed on fish between 6 and 7 days old, unless otherwise noted. All experiments followed institution IACUC protocols as determined by the Harvard University Faculty of Arts and Sciences standing committee on the use of animals in research and teaching.

\section{METHOD DETAILS}

Free-swimming place-preference assay

In order to test whether larval zebrafish avoid salts, we sought to develop a rig that would allow us to determine a larva's place preference within a chemical gradient. Previous studies have examined chemical preference in adult zebrafish by using flow chambers. These strategies, however, introduce two main confounds that we wished to avoid. First, any chemical avoidance behaviors will be convolved with rheotaxis and the optomotor response, and switching chambers forces the larva to behave in opposition to its rheotactic drive. Second, these arenas only create a very steep local gradient at the chamber boundaries, which may not provide the information necessary for the larva to find its preferred location.

Agar pads are made from $3 \%$ low melting point agarose. Agar is poured into premade casts designed to fit the arena. After the agar settles, it is cut out and added to the arena, which is then filled with water, lane by lane. We confirmed the presence of a gradient by using a VAPRO ${ }^{\circledR}$ vapor pressure osmometer (Model \# 5600) to measure the osmolarity of the water in 15-minute intervals at the quartiles of the arena's length while fish were traversing the gradient. After the fish are added, the initial background image is calculated for $20 \mathrm{~s}$ and the experiment begins. To make ionic solutions, salt (all purchased from Sigma-aldrich) was added to the agarose prior to melting. 
Head-embedded chemical stimulation

At present, the designs available for performing calcium imaging of the brains of freely-swimming zebrafish larvae ${ }^{25}$ do not offer the same resolution as generated by traditional methods that require the brain to be immobilized, such as two-photon point scanning and light-sheet microscopy. Therefore, we designed a preparation that would enable simultaneous stimulation of a head-immobilized larva with salt and recording of its behavior. In this preparation, we use gravity to control fluid flow that is directed to the rostral end of the fish by a narrow, $360 \mu \mathrm{m}$ diameter perfusion pencil tip (AutoMate Scientific 04-360). The flow speed was approximately $1.5 \mathrm{ml} /$ minute. Multiple solutions were passed through the perfusion tip via an 8-channel manifold that ensured rapid liquid volume exchange (AutoMate Scientific 04-08-zdv). Solution outputs were regulated by solenoids (Cole Palmer EW-01540-01) via an Arduino ${ }^{\circledR}$ during the experiment such that at all times one and only one solution was being presented to the fish. Presenting a continuous stream of flow both attenuates behavioral responses to sudden changes of flow velocity and hastens the removal of salt at the end of a trial compared to diffusion alone. The dynamics of the pulse were assessed by imaging pulses of $10 \mathrm{nM}$ fluorescein under an epifluorescent microscope (Olympus ${ }^{\circledR}$ MVX10).

\section{Chemical treatments}

For olfactory and lateral line ablations, fish were treated with either 0,2 , or $20 \mu \mathrm{M}$ Copper Sulfate $\left(\mathrm{CuSO}_{4}\right)$. Fish were incubated for one hour in the chemical and then given one hour to recover before behavioral experiments. Ablation of the lateral line was confirmed anatomically by incubating the fish in a dye, FM1-43 (ThermoFisher T3163) for five minutes followed by a 15-minute wash in fish water and taking images under an epifluorescent microscope.

\section{Light-sheet microscopy}

Volumetric imaging experiments were performed with a custom-built single-photon lightsheet microscope similar to that described previously ${ }^{59}$. One difference, however, is that we used a transparent specimen chamber and holder to enable tail tracking via a camera below the fish. For imaging, Nacre - /- larvae positive for GCaMP6s expression under the HuC promoter ${ }^{25}$ were embedded in $3 \%$ agarose. This higher percentage of agarose was used to minimize motion artifacts. To stimulate the fish, we removed the agarose surrounding the nose. To allow behavioral monitoring, we also removed the agarose that surrounds the tail. Larvae were illuminated with a $488 \mathrm{~nm}$ digitally scanned sheet that swept through $200 \mu \mathrm{m}$ of depth with $4 \mu \mathrm{m}$ steps at $1 \mathrm{~Hz}$. During the experiment, fish were stimulated with $10 \mathrm{~s}$ of a given concentration of $\mathrm{NaCl}(25,50,75,100 \mathrm{mM})$ separated by $40 \mathrm{~s}$ of water flow. Each concentration block lasted for 5 minutes and was separated by 5 minutes.

\section{Two-photon microscopy and ablations}

Two-photon microscopy experiments were performed with a custom-built two-photon microscope described previously ${ }^{60}$. Nacre -/- larvae positive for GCaMP6s expression in the brain were embedded in 1.8\% agarose, and the tail and nose were freed as done during light-sheet experiments and embedded behavior experiments. Larvae were imaged with a spectra-physics Mai-tai laser at $950 \mathrm{~nm}$ with $10 \mathrm{~mW}$ of power at the sample. Volumes spanning the olfactory bulb were imaged plane-by-plane at $8 \mu \mathrm{m}$ steps.

For neuromast ablation experiments, the wavelength of the two-photon laser was set to $820 \mathrm{~nm}$. Between 3 and 6 short ( $<100 \mathrm{~ms}$ ) pulses of maximum power laser were delivered to submicron areas of each neuromast until the reported fluorescence became disorganized. For shams, the focal plane was moved $30 \mu \mathrm{m}$ dorsal of the neuromasts and the same net power was delivered.

\section{QUANTIFICATION AND STATISTICAL ANALYSIS}

\section{Behavior analyses}

All analyses were performed with custom-written MATLAB code. Preference indices were calculated based upon the position of the fish along the axis perpendicular to the two agar pads. We define a preference index as the difference in time spent on the half of the arena close to the "test" pad from the time spent away divided by the total length of the experiment:

$$
\text { P.I. }=\frac{t(\text { source })-t(\text { control })}{t(\text { source })+t(\text { control })}
$$

where $t$ (source) and $t$ (control) are the time spent in the half of the arena closest to the salt and control, respectively. As such, the preference index ranges from one for larvae that spend all of their time on the side proximal to the $\mathrm{NaCl}$, and negative one for those that spend all of their time distal to the salt.

To analyze kinematic parameters, bouts were segmented automatically from the absolute speed of the fish combined with identifying periods of high variance in the heading angle. Bouts were then separated by whether they brought the animal closer to or further from the source agarose pad (by at least $0.2 \mathrm{~mm}$ ).

\section{Free swimming simulations}

For simulations, we assumed larvae chose bouts from one of two types of swim events - straight swims and turns. We described the heading angle changed during these swims by a Gaussian, $(0,4.5)$, and lognormal, $\log N(3.5,0.6)$, distribution for straight swims and turns, respectively. For every swim, larvae choose a heading angle turn from a probability distribution that is a linear combination of the above distributions. As the virtual fish navigates, it experiences changes in salt concentrations. We simplify the gradient by 
estimating it as a linear increase from the control agar pad to the source that rises linearly with time. We fit slopes to reach a peak concentration of $10 \%$ of the source at the end of the experiment. Following each bout, we redraw the heading distribution, based upon the change in salt concentration. As the change in salt increases, we apply a higher weight to the turn distribution. This weight is assumed to be a linear function of the change in salt concentration caused by the previous bout. Bouts are assumed to take place at a frequency of $0.8 \mathrm{~Hz}$ and move the fish by $1 \mathrm{~cm}$.

\section{Image Analysis}

After imaging experiments, all data was segmented into activity units that approximate cells. Segmentation was performed using an algorithm based on the one developed by Portugues et $\mathrm{al}^{26}$. After segmentation of units, in order to avoid including motion artifacts during vigorous movements, we removed frames that co-occurred large tail bends during segmentation, and reconstructed the final trace by filling in that gap with a b-spline interpolation generated from the surrounding frames. The eyes, which are heavily autofluorescent, were manually masked out of each stack to avoid segmentation and registration errors. Registration to the reference brain was performed using the Computational Morphometry Toolkit (CMTK; ${ }^{61}$ ) as described previously ${ }^{27}$. The following parameter set was used:

a -w -r 01 -v -T 8 -X 32 -C 6 -G 24 -A '-accuracy 1.6' -W '-accuracy 6.4'

The relationship between the stimulus and each activity unit was determined by calculating their mutual information. To do so, each neural signal was reduced to a 300 block time series, where each block was the average activity from $10 \mathrm{~s}$. This made the score blind to dynamics within the pulse, so, for instance, stereotypic differentiating cells could also be discovered. The resulting signal was normalized to range from 0 to 1 and then binned into 10 equal-sized units. The same was done with the stimulus signal, which was convolved with a $3 \mathrm{~s}$ exponential decay to account for the stimulus and GCaMP6S off-kinetics, which together lead to signal bleeding into the next $10 \mathrm{~s}$ block. A behavioral signal was generated by counting the number of behavioral responses in each $10 \mathrm{~s}$ block. A combined behavior and stimulus signal was generated by multiplying the behavior and stimulus signals. Mutual information was then defined as follows:

$$
\boldsymbol{I}(\boldsymbol{X} ; \boldsymbol{Y})=\sum_{\boldsymbol{X}=1}^{10} \sum_{\boldsymbol{y}=1}^{10} \boldsymbol{P}(\boldsymbol{X} \cap \boldsymbol{Y}) * \ln \left(\frac{\boldsymbol{P}(\boldsymbol{X} \cap \boldsymbol{Y})}{\boldsymbol{P}(\boldsymbol{X}) * \boldsymbol{P}(\boldsymbol{Y})}\right)
$$

Here, $x$ and $y$ represent stimulus and activity bins. Scores were then normalized by the entropy of the stimulus.

To test the classification ability of given regions, support vector machines were trained on a random subset of $50 \%$ of trials to identify from the population activity within that region either the concentration of salt in the trial, or whether the animal responded during the trial. The resulting classifier was then cross-validated with the remaining trials. We report the performance of this cross-validation. For each fish, this process was repeated 50 times and performance was averaged across these repetitions.

For two-photon imaging experiments, we defined units as active based upon their coherence across trials. Namely, we asked for each trial, what is the correlation of the units activity with the average across trials. All units with an average correlation across trials greater than 0.8 were deemed active. This threshold was determined by the value above which the probability of being seen in shuffled data is less than 0.01 . For comparisons across fish, calcium traces were normalized by the variance of the unstimulated activity. 